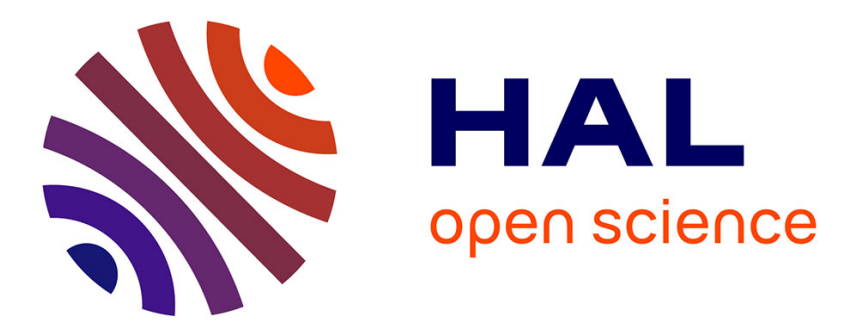

\title{
Bubble-to-void transition promoted by oxide nanoparticles in ODS-EUROFER steel ion implanted to high He content
}

O. Emelyanova, A. Gentils, V.A. Borodin, M.G. Ganchenkova, P.V.

Vladimirov, P.S. Dzhumaev, I.A. Golovchanskiy, R. Lindau, A. Möslang

\section{To cite this version:}

O. Emelyanova, A. Gentils, V.A. Borodin, M.G. Ganchenkova, P.V. Vladimirov, et al.. Bubble-to-void transition promoted by oxide nanoparticles in ODS-EUROFER steel ion implanted to high He content. Journal of Nuclear Materials, 2021, 545, pp.152724. 10.1016/j.jnucmat.2020.152724 . hal-03098909

\section{HAL Id: hal-03098909 \\ https://hal.science/hal-03098909}

Submitted on 24 Aug 2021

HAL is a multi-disciplinary open access archive for the deposit and dissemination of scientific research documents, whether they are published or not. The documents may come from teaching and research institutions in France or abroad, or from public or private research centers.
L'archive ouverte pluridisciplinaire HAL, est destinée au dépôt et à la diffusion de documents scientifiques de niveau recherche, publiés ou non, émanant des établissements d'enseignement et de recherche français ou étrangers, des laboratoires publics ou privés. 
Bubble-to-void transition promoted by oxide nanoparticles in ODS-EUROFER steel ion implanted to high He content

O. Emelyanova ${ }^{1,2}$, A. Gentils ${ }^{1}$, V.A. Borodin ${ }^{2,3}$, M.G. Ganchenkova ${ }^{2}$, P.V. Vladimirov ${ }^{3,4}$, P.S. Dzhumaev $^{2}$, I.A. Golovchanskiy ${ }^{5,6}$, R. Lindau ${ }^{4}$, A. Möslang ${ }^{4}$

${ }^{1}$ Université Paris-Saclay, CNRS/IN2P3, IJCLab, 91405 Orsay, France

${ }^{2}$ National Research Nuclear University MEPhI, 115409 Moscow, Russia

${ }^{3}$ National Research Centre 'Kurchatov institute',123182 Moscow, Russia

${ }^{4}$ IAM-AWP, Karlsruhe Institute of Technology, D-76344, Eggenstein-Leopoldshafen, Germany

${ }^{5}$ National University of Science and Technology MISiS, 119049 Moscow, Russia

${ }^{6}$ Moscow Institute of Physics and Technology, 141700 Dolgoprudny, Russia

The paper deals with a detailed study of He-filled cavity ensemble development in ODSEurofer steel implanted with $10 \mathrm{keV}$ helium ions to a high peak concentration of $8.5 \times 10^{3}$ appm both with and without simultaneous irradiation with $4 \mathrm{MeV}$ gold ions, which allowed us to strongly vary the ratios of $\mathrm{dpa} / \mathrm{He}$ introduction. The subsequent transmission electron microscopy examination reveals excellent radiation stability of He-implanted sample in the single-beam implantation mode. In contrast, after the simultaneous dual-beam irradiation the occurrence of a bubble-to-void transition was observed for bubbles that were associated with yttria nanoparticles. The relative importance of different He bubble families observed in the He-implanted samples for the swelling accumulation is quantitatively assessed, emphasizing the potential risks of abrupt swelling acceleration in the case of bubble-to-void transition launched by nanoparticles. A model of bubble-to-void transition for gas bubbles associated with spherical second-phase particles is developed and used to rationalize experimental observations.

Key words: ODS-Eurofer steel, ion implantation, TEM, helium, cavities, swelling, bubble-to-void transition, modeling

\section{Corresponding author:}

O. Emelyanova

e-mail: olga.emelianova@ijclab.in2p3.fr,ovemelyanova@mephi.ru 


\section{Introduction}

One of the challenges for the development of future nuclear facilities, such as fusion and the next generation (GenIV) of fission reactors, is the selection of appropriate structural materials for their active zones. Radiation environment in these facilities is expected to be much more severe than in modern nuclear reactors owing to high operation temperatures $\left(>650^{\circ} \mathrm{C}\right)$, high damage levels, as well as high rates of light gas accumulation [1]. Among promising candidates for the use in such conditions are reduced-activation ferritic-martensitic oxide-dispersion-strengthened (RAFM ODS) steels. Saturation of steel with high number densities of nanosize metal-oxide particles not only improves its high-temperature mechanical stability [2-4], but is also expected to improve radiation resistance $[3,4]$.

The beneficial role of oxide nanoparticles is commonly attributed to their ability to act as nucleation centers for small helium bubbles [4,5]. An artificial increase of the bubble number density efficiently decreases the average bubble size and thus prevents or strongly postpones the conversion of bubbles into a population of freely growing vacancy voids that promote nonsaturable swelling at quite fast rates, of the order of percent per dpa $[3,6]$. In the context of the paper we differentiate between gas bubbles and voids in the sense suggested in Ref. [7], treating gas-filled cavitiesstabilized by their internal gas pressure as bubbles, while cavities which are primarily agglomerations of vacancies, even if they contain some number of gas atoms, as voids. While the common reasoning described above sound reasonable, the true efficiency of bubble nucleation on oxide particles as a method of swelling mitigation is not fully obvious.

Indeed, the suppression of bubble-to-void conversion can be achieved in different ways, either by increasing the critical size that the bubbles should reach for such a transition, or by decreasing the sizes of helium bubbles themselves. On the one hand, the critical bubble size for the bubble-to-void transition is known to be inversely proportional to the effective vacancy supersaturation in the material [8], the latter being determined by the balance between irradiation intensity and the efficiency of point defect consumption at different sinks. An additional population of bubbles on oxide particles increases the total sink strength for point defects and thus contributes to the increase of the critical transition size. However, the relative importance of this increase is not evident because ODS steels are very complex materials with high densities of other possible point defect sinks. In the tempered ODS-Eurofer, in particular, these sinks include dislocations, grain boundaries and carbide precipitates $\left(\mathrm{M}_{23} \mathrm{C}_{6}\right)$ [2,9-13]. On the other hand, while helium bubbles in ODS steels remain small even at quite high levels of accumulated helium, they are known to be associated not only with ODS particles, but with the other microstructural defects as well, and the bubble families on different microstructural defects can have different characteristic sizes [14-16]. Evidently, the bubble family with the largest typical size has the 
largest chances to reach the critical size first. From this point of view, bubbles on second phase precipitates are in the group of risk because such bubbles require less gas atoms to reach any predefined diameter than bubbles in the bulk [17].

It remains an open question, whether the bubble-to-void transition is a potential issue for ODS steels in the next generation of fusion and fission facilities, but it is instructive to verify, whether one can procure sufficiently reasonable conditions to demonstrate the effect on the modern equipment. In the current literature one can find only a few reports $[18,19]$ mentioning bubble-tovoid transition in ODS alloys PM2000 and 14YW that had accumulated more than a thousand appm He after neutron irradiation to $>20 \mathrm{dpa}$ at $500^{\circ} \mathrm{C}$. Importantly, the large cavities, interpreted by the authors as voids, were always associated with nanoparticles, which motivates a more detailed study of the role of particles in the acceleration of swelling onset in ODS steels, the mechanisms involved in the effect, and the conditions required for its manifestation.

A quest for bubble-to-void transition in ODS steels implies at least two conditions. First of all, one should accumulate sufficiently high concentrations of helium in order to force the bubbles to grow sufficiently large in spite of the higher bubble number densities and thus reduced bubbles sizes promoted by oxide particles in ODS ferritic-martensitic steels as compared to their non-ODS counterparts. The experiments observing bi-modal cavity distribution in non-ODS ferriticmartensitic steels [20-24] indicate, however, that the critical diameter required for bubble conversion into voids is not very large (presumably $\sim 4-5 \mathrm{~nm}$ ) and so the required He content in steel should be achievable, even though quite high (at the level of at least thousands appm, as indicated by the above mentioned Refs. $[18,19])$. Second, one should decrease as low as possible the critical transition size, which can be achieved by using high point defect generation rates. The need to satisfy both demands simultaneously suggests ion implantation experiment as an attractive option.

In this work, we report a detailed study of the He-filled cavity ensemble development in ODS-Eurofer steel implanted in a fast ion accelerator with helium ions to a very high peak concentration in two regimes, involving low and high ratios of $\mathrm{dpa} / \mathrm{He}$ introduction. A considerable increase of the dpa/He ratio is achieved by simultaneous use of a secondary energetic gold ion beam. The results of subsequent transmission electron microscopy (TEM) examination, reported in sect. 3, demonstrate quite similar development of He-filled cavity ensembles in both irradiation modes with the only exception that bubble-to-void transition is observed for the mode with high $\mathrm{dpa} / \mathrm{He}$ introduction ratio. A quantitative theoretical model of bubble-to-void transition for cavities on spherical second-phase particles is developed in sect. 4 and used to rationalize experimental observations in sect.5. 


\section{Experimental details}

\subsection{Material and irradiation}

ODS-Eurofer used in this study is an oxide-dispersion strengthened ferritic-martensitic steel developed in Karlsruhe Institute of Technology (KIT) in cooperation with Plansee AG (Austria). The basic composition of ODS-Eurofer steel is $9 \mathrm{Cr}-1 \mathrm{~W}-0.08 \mathrm{Ta}-0.2 \mathrm{~V}-0.07 \mathrm{C}-0.4 \mathrm{Mn}-$ $0.3 \mathrm{Y}_{2} \mathrm{O}_{3}$ (wt.\%). The steel was produced using powder metallurgy technique that included inert gas atomization of EUROFER 97, mechanical alloying in industrial ball mills of attritor type, followed by hot isostatic pressing and hot rolling in the austenitic temperature range. The investigated steel samples were supplied in the tempered condition, i.e. after finishing rolling at the temperature of $980{ }^{\circ} \mathrm{C}$ followed by tempering at $750{ }^{\circ} \mathrm{C}$ for $2 \mathrm{~h}[2]$.

Slices of ODS-Eurofer steel with the thickness of $\sim 300 \mu \mathrm{m}$ were cut out, mechanically grinded down to $\sim 100 \mu \mathrm{m}$ and, finally, the discs of $3 \mathrm{~mm}$ diameter were punched out from the slices. Prior to irradiation, the discs were electropolished in a StruersTenupol-5 unit with a 10\% $\mathrm{HClO}_{4}+90 \% \mathrm{CH}_{3} \mathrm{OH}$ solution at $-20{ }^{\circ} \mathrm{C}$ in order to remove any damage due to mechanical polishing (for samples used in single-beam implantation) or to prepare electron transparent samples for dual-beam in situ implantation. After electropolishing, the samples were cleaned from both sides by ion milling system PIPS 693 using a $3 \mathrm{eV}$ ion beam and 5-6 etching angle for 2 minutes.

The irradiation of ODS-Eurofer steel was performed at the JANNuS-Orsay/SCALP facility of the IJCLab (Orsay, France) [25] at $823 \mathrm{~K}$, using two regimes:

(1) Dual-beam regime that involved simultaneous in situ dual beam irradiation (by means of coupling $190 \mathrm{kV}$ ion implanter IRMA, $2 \mathrm{MV}$ tandem accelerator ARAMIS and transmission electron microscope) of electron transparent $3 \mathrm{~mm}$ disk with $10 \mathrm{keV} \mathrm{He}^{+}$ions with the flux of $5 \times 10^{11} \mathrm{~cm}^{-2} \mathrm{~s}^{-1}$ to the fluence of $5 \times 10^{15} \mathrm{~cm}^{-2}$, and $4 \mathrm{MeV} \mathrm{Au}{ }^{2+}$ ions with the flux of $4 \times 10^{11} \mathrm{~cm}^{-2} \mathrm{~s}^{-1}$ to the fluence of $4.5 \times 10^{15} \mathrm{~cm}^{-2}$;

(2) Single beam regime that involved ex situ irradiation of $100 \mu \mathrm{m}$ thick disk on ion implanter with $10 \mathrm{keV} \mathrm{He}$ ions with the flux of $5 \times 10^{11} \mathrm{~cm}^{-2} \mathrm{~s}^{-1}$ to the fluence of $5 \times 10^{15} \mathrm{~cm}^{-2}$.

As can be noticed, the $\mathrm{He}^{+}$ion beam parameters were identical in both regimes, while the difference was in the presence of the gold ion beam that was used in order to strongly increase the efficiency of radiation damage production and, thus, the $\mathrm{dpa} / \mathrm{He}$ introduction ratio. Due to the geometry of the facility, the thin foils were tilted off of the optical axis so that both the $\mathrm{Au}^{2+}$ and $\mathrm{He}^{+}$ion beams made an angle of $23^{\circ}$ with the sample surface normal direction. The energy of $\mathrm{Au}^{2+}$ ions was selected to be sufficiently high in order to minimize sputtering and the influence of injected interstitials on bubble nucleation in the region of interest (ROI) [26,27], which in our case 
falls from the sample surface to the depth $\sim 40-60 \mathrm{~nm}$, i.e. the thickness transparent for TEM. The selected $\mathrm{He}+$ ion energy of $10 \mathrm{keV}$ was preliminary verified to produce relatively uniform cavity size distribution over ROI, while the cavity number density depth variation roughly followed the implanted helium profile with no visible influence of sample free surfaces. Unfortunately, no direct TEM observations during the ion implantation were possible because TEM objective lens had to be switched off during the in situ experiment in order to prevent deflection of low-energy He ions coming from the implanter beam line. Therefore, TEM observations in this implantation regime were done only when the irradiation was over.

The damage and He concentration profiles for simultaneous in situ dual beam irradiation predicted by calculations with the code SRIM-2008 [28] are shown in Fig.1(a). The calculations assumed the matrix atom displacement energy of $40 \mathrm{eV}$ and the full damage cascade calculation method. The predicted primary damage generation rate from both $\mathrm{Au}^{2+}$ and $\mathrm{He}^{+}$ion beams in the peak region of He accumulation ( $\sim 44 \mathrm{~nm}$ from the beam-facing surface) was predicted to equal $2.4 \times 10^{-3} \mathrm{dpa} / \mathrm{s}$, so that the total number of displacements during the whole irradiation reached $\sim 26.9$ dpa. The helium accumulation rate was about $0.85 \mathrm{appm} / \mathrm{s}$, so that the estimated He concentration in the peak by the end of irradiation reached $\sim 8.5 \times 10^{3} \mathrm{appm}$. Thus, the average ratio of dpa production to He accumulation in the region of interest was $\sim 3 \times 10^{-3} \mathrm{dpa} / \mathrm{appm}$. $<$ Fig.1 $>$

In the single beam regime, the sample of ODS-Eurofer steel was implanted with $10 \mathrm{keV}$ $\mathrm{He}^{+}$ions falling normally to the sample surface. The damage and He concentration profiles for single beam irradiation predicted by SRIM based calculations are shown in Fig. 1(b). Since $\mathrm{He}^{+}$ ion flux and fluence were the same as for the dual beam implantation experiment, the same $\mathrm{He}$ accumulation rates and final $\mathrm{He}$ concentrations were achieved, but the predicted damage generation rate was only $3.9 \times 10^{-5} \mathrm{dpa} / \mathrm{s}$ and the total number of displacements was $0.39 \mathrm{dpa}$. Hence, the average ratio of damage production to He accumulation was in this case only $5 \times 10^{-5}$ dpa/appm.

\subsection{TEM data accumulation and processing for swelling estimation}

After dual-beam irradiation, the sample of ODS-Eurofer was cleaned from both sides by ion milling system PIPS 693 using a $1 \mathrm{keV}$ ion beam and $4-5^{\circ}$ etching angle for 2 minutes and then directly used for transmission electron microscopy (TEM) investigations. After the single-beam irradiation, the samples were additionally thinned from the unirradiated side to the electron transparency using Tenupol-5 unit with the same electrolyte and thinning regime as those used prior to ion implantation. Electropolished samples were cleaned by ion beam etching in the same way as dual-beam irradiated samples. 
TEM investigations were performed using a FEI Tecnai $\mathrm{G}^{2}$ microscope with $\mathrm{LaB}_{6}$ filament equipped with a Gatan image filter for EELS measurements. Among the three techniques typically used to produce cavity images, namely - the trough-focal series method in a conventional bright field (BF) TEM, scanning TEM in bright field (BF STEM) and annular dark-field (ADF or HAADF) modes [29] or Aberration-Corrected X-Ray Spectrum Imaging [30], we have selected BF TEM trough-focal series method as the most straightforward one that allows rapid processing of large data arrays. In order to estimate the number density of cavities, the local thickness of TEM samples was measured by EELS log-ratio approach, which causes an uncertainty of $\pm 10 \%[31,32]$.

Transmission electron microscopy is a well-established technique for the swelling estimation based on the parameters of visible cavity populations. In the approach prescribed by ASTM standard [33], the swelling, $S_{\mathrm{ASTM}}$, is defined in terms of the cumulative volume $\sum_{i} V_{c i}$ of all cavities in the visible area,

$$
S_{\mathrm{ASTM}}=<\sum_{i} V_{c i} / V>\times 100 \%
$$

where $V$ is the volume of the visible area. However, in the case of He implantation into ODS steel, which results in several different cavity families associated with different microstructural components of the steel, this approach is insufficient because it doesn't allow to figure out the contributions of each cavity family to the cumulative swelling. For this reason, here we evaluate the cumulative swelling, $S$, by an indirect approach as:

$$
S=\sum_{k} S^{k}
$$

where $S^{k}=V_{c}^{k} \cdot N_{c}^{k} \cdot 100 \%$ is the swelling value associated with a particular cavity family denoted with superscript $k$ (e.g. $k=\mathrm{V}$ for cavities in the defect-free grain volume and $k=\mathrm{D}, \mathrm{GB}$, and $\mathrm{P}$ for cavities on dislocations, grain boundaries, and oxide particles, respectively), $V_{c}^{k}$ and $N_{c}^{k}$ are the average volume and number density of bubbles in the $k$-th family type.

The average cavity volume for the cavities located in the grain matrix and associated with extended defects (dislocations and grain boundaries) is calculated as:

$$
V_{c}^{k}=\frac{\pi}{6} \cdot\left\langle D_{c}^{k}\right\rangle^{3}
$$

where $\left\langle D_{c}^{k}\right\rangle$ is the effective bubble diameter obtained from a statistical analysis of the bubbles associated with each microstructural component.

The cavities attached to $\mathrm{Y}_{2} \mathrm{O}_{3}$ nanoparticles in ODS steels typically have a specific lenslike shape [34], which is also true for the current study, see Fig. 2(a). So the cavity volume is noticeably less than that predicted by equation (3.1). For this reason, the volumes of cavities attached to oxide nanoparticles were calculated as: 


$$
V_{c}^{p}=\frac{\pi}{3}\left[R^{3}(2+\cos (\alpha+\beta))(1-\cos (\alpha+\beta))^{2}-R_{p}^{3}(2+\cos \beta)(1-\cos \beta)^{2}\right],
$$

where $R$ is the cavity radius, $R_{p}$ is the $\mathrm{Y}_{2} \mathrm{O}_{3}$ nanoparticle radius, and the angles $\alpha$ and $\beta$ are as defined in Fig. 2(b).

$<$ Fig. 2>

The wetting angle $\alpha$ for equilibrium cavities is determined by the surface tension on the interfaces that meet at the cavity rim [35],

$$
\cos \alpha=\frac{\gamma_{s m}-\gamma_{s c}}{\gamma}
$$

where $\gamma_{s m}$ and $\gamma_{s c}$ stand for the specific surface energies of substrate interfaces with the matrix and the vacuum, respectively, while $\gamma$ is the surface tension for the cavity-matrix interface. Parameter $\alpha$ is thus fully determined by material properties. The direct measurements on TEM images (such as that shown in Fig. 2(a)) has confirmed that in our case the wetting angle is practically insensitive to either the nanoparticle size or implantation conditions and equals to $48 \pm 5^{\circ}$. The cosines entering equation (3.2) can be expressed in terms of $R, R_{p}$, and $\alpha$ as (see Appendix):

$$
\cos (\beta)=\frac{\left(R_{p}-R \cos (\alpha)\right)}{\sqrt{R_{p}^{2}-2 R R_{p} \cos (\alpha)+R^{2}}} ; \quad \cos (\alpha+\beta)=\frac{\left(R_{p} \cos (\alpha)-R\right)}{\sqrt{R_{p}^{2}-2 R R_{p} \cos (\alpha)+R^{2}}} .
$$

In order to estimate the contributions of different families of He filled cavities to overall swelling, one must know not only the average cavity sizes that are directly measurable in TEM images, but also the cavity number densities per unit volume. However, only the number density of cavities in the grain matrix can be estimated directly from TEM images. For cavities on extended defects, reliable estimates can be obtained only for specific bubble number densities, $\left\langle N_{c}^{k}\right\rangle$, such as the bubble number density per unit dislocation length or per unit grain boundary surface. In order to convert specific number densities into those normalized per unit sample volume, the following relation was used

$$
N_{V}^{k}=\left\langle N_{c}^{k}\right\rangle \cdot N_{k},
$$

where $N_{k}$ is the average density of relevant extended defects per unit material volume typical for the studied material. The average volumetric densities of dislocations can be taken from the earlier studies of non-irradiated ODS-Eurofer [2,9-13,36]. However, to the best of our knowledge, the volumetric density of grain boundaries required for the swelling calculations has not been reported in the literature. In this study, the density of grain boundaries was estimated using EBSD measurements on unirradiated samples using the Oxford Instruments CHANNEL 5 EBSD system 
installed on EVO XVP SEM microscope. Only high angle grain boundaries with misorientation angle $>10^{\circ}$ were considered. Finally, for the bubbles on oxide particles we can rely on the observation that each oxide particle contains typically a single cavity, so that the volumetric number density of this cavity population coincides with the typical number density of yttria particles in ODS-Eurofer. The typical parameters for microstructural features used below to estimate the swelling contributions from different bubble families are summarized in Table 1. $<$ Table $1>$

Strictly speaking, in addition to nanosize oxide particles, ODS-Eurofer contains also relatively large particles of $\mathrm{M}_{23} \mathrm{C}_{6}$ carbide that are also potentially able to promote He-filled cavity formation [37]. However, their number density is much lower than that of oxide particles and the electron transparent zones of the studied TEM samples contained no visible carbides. So, a potential contribution to swelling from bubbles located on carbide precipitates is not taken into account here.

\section{Experimental observations}

\subsection{Microstructural analysis}

Typical BF TEM trough-focus images of different zones on ODS-Eurofer steel sample subjected to in situ simultaneous dual-beam $\mathrm{He}^{+}+\mathrm{Au}^{2+}$ irradiation and to single-beam $\mathrm{He}^{+}$ion implantation at $823 \mathrm{~K}$ are shown in Figs.3(a,c) and 3(b,d), respectively. $<$ Fig3 $>$

As can be noticed in Fig. 3, the qualitative patterns of helium filled cavity development are largely similar in both irradiation regimes. He filled cavities are met not only in the grain bulk, but decorate grain boundaries, dislocations, and yttria nanoparticles as well. In fact, all visible oxide nanoparticles are decorated with cavities, hosting mostly a single cavity per particle, or, in very rare cases, two cavities. As a result, cavities are non-uniformly distributed in space and follow local variations in associated microstructure. The dislocations themselves are not visible in the imaging conditions optimized for cavity observation. Within each of both irradiation regimes, populations of He-filled cavities located in the grain bulk and associated with extended defects (grain boundaries and dislocations) demonstrate quite similar bubble sizes, while the cavities attached to oxide nanoparticles are notably larger than those in the other bubble families. At the same time, the accelerated defect production provided by the secondary $\mathrm{Au}^{2+}$ beam results in the increase of the average cavity sizes for all described bubble families as compared to single-beam regime. Moreover, as can be noticed in Fig.3(c), after the dual beam irradiation some $\mathrm{Y}_{2} \mathrm{O}_{3}$ nanoparticles are associated with cavities at least 3 times larger than cavities on other microstructural components. This effect was not observed after single-beam $\mathrm{He}^{+}$implantation. For 
a clearer view, BF TEM images of He cavities of different sizes attached to nano-oxides in ODSEurofer sample after dual beam $\mathrm{He}^{+}+\mathrm{Au}^{+}$irradiation are shown in Fig. 4 at a higher magnification. $<$ Fig4 $>$

It has been found that the size of a cavity attached to an $\mathrm{Y}_{2} \mathrm{O}_{3}$ particle correlates with the size of the host particle in both irradiation regimes; the larger the oxide particle, the larger is the associated cavity. A similar effect has been reported in the literature for steels containing nanoparticles of other oxide compositions (e.g. $\mathrm{ZrO}_{2}, \mathrm{HfO}_{2}$ and $\mathrm{Y}_{2} \mathrm{TiO}_{7}[38,39]$ ) and seems to be a general trend for He bubbles on oxide particles. To characterize the effect in quantitative terms, the measured diameters $D_{c}^{p}$ of cavities on oxide particles are plotted in Fig. 5 versus their host particle diameters $D_{p}$ along with appropriate power law trend lines. The above mentioned general trend is clearly visible for both the dual-beam $\mathrm{He}^{+}+\mathrm{Au}^{2+}$ irradiation and the single-beam $\mathrm{He}^{+}$ implantation.

$<$ Fig5 $>$

As can be seen in Fig. 5, in the single-beam $\mathrm{He}^{+}$implantation regime the data are nicely described by a power law of the form $D_{c}^{p}\left(D_{p}\right)=a \cdot D_{p}{ }^{b}$. The best fit line for this regime (blue trend line in Fig. 5) is achieved for the fitting parameters $a=1.55$ and $b=0.66$. The absolute majority of bubbles have smaller sizes than those of the host particles $\left(R<R_{p}\right)$, falling in the area shaded gray in Fig. 5.

In contrast, after the dual-beam irradiation the cavities on yttria particles fall into two different subfamilies. For the most part, the cavities are smaller or comparable in size with the host precipitates, like in the case of single-beam implantation. The trend line for this subfamily is described by the power low with the same value of $b$ and with a slightly larger $a=1.67$.

But there is also a group of 'large' cavities that strongly deviate from the single-beam trend line, having the sizes well above that of the host oxide particle. Defining (rather arbitrarily) the 'large' cavities as those with the diameter exceeding that of the host particle (i.e. $D_{c}^{p}>D_{p}$ ), the dependence $D_{c}^{p}\left(D_{p}\right)$ can be roughly fitted by the power law with $a=1.55$ and $b=1.02$ (red trend line in Fig. 5).

If we define the average size of cavities on oxide particles as the value predicted by the best fit power law for the average particle size in the steel (in our case $\left\langle D_{p}\right\rangle=12 \mathrm{~nm}$, see Table 1), then the average cavity size for single-beam $\mathrm{He}^{+}$implantation, $\left\langle D_{c}^{p}\right\rangle=8.1 \mathrm{~nm}$, is only slightly smaller than that for 'small' cavity population in the dual-beam irradiation case, $\left\langle D_{c}^{p}\right\rangle=8.7 \mathrm{~nm}$. 
But the average size for the 'large' cavity population formed during the dual-beam irradiation is noticeably larger, $\left\langle D_{c}^{p}\right\rangle=19.5 \mathrm{~nm}$.

Summing up, a typical bi-dimensional cavity size distribution for the cavities attached to yttria particles is observed after dual-beam $\mathrm{He}^{+}+\mathrm{Au}^{+}$irradiation. Such kind of size distribution is not uncommon for steels irradiated in conditions characterized by high dpa/He ratios and is usually interpreted as a result of the so called bubble-to-void transition [42]. This means that the cavities that have accumulated a certain critical number of gas atoms do not need more gas in order to grow, increasing their size in a void-like manner by directly collecting radiation-generated vacancies. Usually only a small part of the whole cavity population undergoes the bubble-to-void transition. If we accept that this effect is responsible for our observations, the 'large' cavities can be treated as 'voids' in the sense that their sizes are no more related to the number of gas atoms they contain, but are rather determined by the level of radiation damage created by irradiation. The remaining 'small' cavities can be treated as nearly equilibrium gas bubbles. As can be noticed in Fig. 5, the 'void' population is associated with relatively large particles. This observation gives indirect evidence in support of the bubble-to-void transition occurrence in our case, as will be discussed in sect. 5 below.

\subsection{Estimated contributions of different bubble populations to the swelling of ODS-Eurofer steel}

In order to estimate the relative contributions of different cavity families to swelling and to clarify a potential modification of the role of bubble-associated cavities that have undergone a 'small' to 'large' transition, we have collected quantitative estimates of the parameters of all cavity families and their contributions to expected swelling in Table 2. For clarity, the data are also drawn in Fig. 6 for single-beam and dual-beam regimes in parallel.

$<$ Table2>

$<$ Fig6 $>$

As can be seen, within each sample there is relatively little difference in size between helium filled cavities in the bulk, on dislocations and at the grain boundaries (within $\sim 10-20 \%$ ). In both samples, cavities on yttria particles are the largest ones. At the same time, in terms of volumetric number density, the largest shares of the whole cavity population in both irradiation regimes are for cavities in the bulk and at the grain boundaries, whereas dislocations and, especially, oxide nanoparticles provide lower contributions.

A comparison between two irradiation regimes presented in Fig. 6 clearly shows that an additional damage production by $\mathrm{Au}^{2+}$ ions systematically reduces the number density and increases the average sizes of bubbles located in the bulk and on extended defects. However, if one does not take into account the large cavities on oxide particles, the differences between the 
dual-beam and single beam irradiations are quite moderate. The most strongly affected are grain boundaries, where the additional damage production results in the increase of the average bubble size to $\left\langle D_{c}^{G B}\right\rangle=7.1 \mathrm{~nm}$, as compared to $\left\langle D_{c}^{G B}\right\rangle=4.8 \mathrm{~nm}$ in the single-beam implantation regime. In fact, only the appearance of large cavities (voids) on yttria particles constitutes a remarkable qualitative difference between the dual-beam irradiation and the single-beam He implantation.

In terms of swelling estimation, the presence of two populations of cavities on yttria particles in the dual-beam irradiation regime makes the estimates of their contributions to swelling uncertain because of the poor statistics of large cavities and the impossibility to find out which part of bubbles has undergone bubble-to-void transition. Therefore, in the subsequent analysis the swelling values associated with oxide nanoparticles are calculated for two limiting cases, either completely neglecting 'large' cavities, or assuming that all bubbles on oxide particles undergo the bubble-to-void transition. The latter assumption is obviously invalid in our conditions; it is used only to estimate the possible upper bound for swelling related to cavities on oxide particles.

As can be seen, the addition of a secondary $\mathrm{Au}^{2+}$ beam increases contributions to swelling for all cavity populations, either in the bulk, or on microstructural defects. The magnitude of the increase is not very large, however, if one does not consider large cavities on oxide particles. The strongest effect is observed for He cavities in the bulk; their contribution to swelling increases approximately twice. Overall, if large voids on oxide particles are not taken into account, the effect of accelerated damage on swelling is very moderate and, among the microstructural defects, the largest contribution is provided by grain boundary cavity population, just as is the case in the single-beam helium implantation regime.

However, the creation of large voids on oxide nanoparticles, if it indeed can be ascribed to the bubble-to-void-transition, changes the situation with swelling drastically, as illustrated in Fig.6(d). When neglecting the contribution of large voids, the swelling in the dual-beam irradiation case is only 1.7 times larger compared to the case of single-beam helium implantation. In case where all the bubbles associated with nanoparticles would undergo such a transition, their contribution to swelling would increase by an order of magnitude, to $3.3 \%$, i.e. well above the contribution of any other cavity family. The expected cumulative swelling would then be higher than the single-beam $\mathrm{He}^{+}$implantation relevant value by a factor of 6.65. In reality, the swelling value for dual-beam irradiation conditions falls somewhere between the two limiting values. But one should not forget that with the increase of irradiation dose the swelling contribution from the large voids grow much faster than could be provided by all He bubbles populations because the growth of large voids is no longer controlled by the number of helium atoms they retain. Therefore it is clear that bubble-to-void transition qualitatively changes steel resistance to swelling. 


\section{The mechanism of bubble-to-void transition acceleration by oxide nanoparticles}

The results of dual-beam $\mathrm{He}^{+}+\mathrm{Au}^{2+}$ ion irradiation demonstrate that steel saturation with ODS oxide particles results in the creation of large cavities that are not observed in the case of single-beam He irradiation. Having in mind that the main difference between these regimes is the much higher $\mathrm{dpa} / \mathrm{He}$ introduction ratio in the case of double-beam irradiation, it has been assumed that the appearance of large cavities resulted from bubble-to-void transition, which is known to be simplified by the increase of vacancy supersaturation in the irradiated material [8]. A specific feature of the observed large cavities was their attachment to oxide nanoparticles, which indicates that oxide particles might trigger the bubble-to-void transition. The physical reasons for this may be twofold. First of all, a cavity-associated bubble has smaller volume as compared to a bubble of the same size in the bulk and thus requires less gas atoms to reach the pressure, needed for the transition. Second, it was experimentally observed that the effective diameters of bubbles on oxide particles are generally larger than those of bubbles in the bulk or bubbles associated with extended microstructural defects, such as dislocations or grain boundaries. This might also accelerate the achievement of a critical size for the bubble-to-void transition, provided the critical transition size for bubbles on particles is not too different from that for bubbles in the bulk. In this section we suggest a simple quantitative description of the bubble-to-void transition for He bubbles associated with spherical particles. Having in mind the experimental picture, we assume that a particle hosts only a single bubble. In addition, we assume that the cavity is formed on the matrix side of the particle/matrix interface and has approximately spherical form. The latter is not exactly true for the experimental observation above (the voids are typically faceted), but can be considered as a reasonable approximation to simplify the calculations without strongly distorting the expected trends.

\subsection{The basics of bubble-to-void transition theory}

Theoretical description of large-scale irradiation-induced effects in structural materials (such as swelling and irradiation creep) is commonly done in the framework of the so called kinetic rate theory $[43,44]$, which is a version of the mean-field statistical description of a many body problem, involving multiple point defect sinks (voids and/or gas bubbles, precipitates, dislocations, grain boundaries) interacting via diffusional transport of point defects (vacancies and self-interstitials) created in materials by irradiation [45]. Within this formalism, the equation of cavity growth rate (that is the rate of cavity volume, $V$, change with time $t$ ) is commonly written down in the from [46],

$$
\frac{d V}{d t}=\left[Y_{v} D_{v}\left(C_{v}-C_{v}^{\text {th }}\right)-Y_{i} D_{i} C_{i}\right]
$$


where $D_{m}$ and $C_{m}$ are the diffusion coefficients and the mean-field ('average') concentrations of point defects of type $m$ ( $m=v$ or $i$ for vacancies or interstitials, respectively), $C_{v}^{\text {th }}$ is the equilibrium vacancy concentration at the cavity surface and $Y_{m}$ are numerical coefficients, commonly referred to as 'bias factors'. By definition, bias factors of point-defect sinks are properties of sinks and are insensitive to the mean-field point defect concentrations [45]. In turn, the mean-field point defect concentrations are not sensitive to properties of individual point defect sinks, being determined by the balance between the efficiency of point defect generation by irradiation and the full point defect loss efficiency on the whole ensemble of point defect sinks present in the material.

In the simplest case of a spherical cavity with radius $R$ in the bulk, the cavity bias factors have a simple form, $Y_{i}=Y_{v}=4 \pi R$ [43], but in more general cases, they can be quite complicated functions of both cavity size and additional parameters (see e.g. Ref. [47] and references therein). For example, when cavities contain noticeable amount of captured gas atoms, such additional parameters can include internal gas pressure and the elastic properties of point defects and material itself. When cavities are associated with other microstructural defects, such as dislocations, grain boundaries, or precipitates, the cavity bias factors can be additionally modified by the host structural defect assistance to the point defect transport to cavities and thus be very different for vacancies and interstitials. However, in order to explain the reasons for the bubble-to-void transition, it is sufficient to restrict ourselves to the simplest form of bias factors, as suggested in the original paper [8] that has introduced the concept of bubble-to-void transition.

Equation (4.1) can be rewritten as

$$
\frac{d V}{d t}=Y_{v} D_{v} C_{v 0}^{t h}\left(\Delta-\Delta_{v}^{t h}\right)
$$

where $\Delta$ is the effective vacancy supersaturation, defined as

$$
\Delta=\frac{Y_{v} D_{v} C_{v}-Y_{i} D_{i} C_{i}}{Y_{v} D_{v} C_{v 0}^{t h}},
$$

$\Delta_{v}^{t h}=C_{v}^{t h} / C_{v 0}^{t h}, C_{v 0}^{t h}=\exp \left(-E_{v}^{f} / k_{B} T\right)$ is the vacancy concentration in equilibrium with a flat material surface, $E_{v}^{f}$ - the vacancy formation energy in the bulk, $k_{B}$ - the Boltzmann constant and $T$ - the absolute temperature. The growth or shrinkage of an individual cavity is thus determined by the relation between the effective vacancy supersaturation and $\Delta_{v}^{\text {th }}$.

For the simplest case of a cavity without internal gas (i.e. void) it is generally true that its growth rate remains negative for all void sizes smaller than a certain critical value. For a spherical void, this statement can be reformulated so that, in order for a void to grow, its radius $R$ should exceed a certain critical value, $R_{c}$. Then the void grows permanently, provided $\Delta$ does not fall 
down with time. In order to demonstrate this, let us set $Y_{i}=Y_{v}$, so that the effective vacancy supersaturation does not depend on void size, while $\Delta_{v}^{\text {th }}$ is described by the Gibbs-Thomson equation,

$$
\Delta_{v}^{t h}=\exp \left(-\frac{\Omega \sigma_{0}}{k_{B} T}\right),
$$

where $\Omega$ is the atomic volume in the matrix and $\sigma_{0}$ is the surface traction that can be expressed in terms of specific free surface energy (surface tension), $\gamma$, as

$$
\sigma_{0}=-\frac{2 \gamma}{R}
$$

It can be easily verified, that the difference $\Delta-\Delta_{v}^{\text {th }}$ monotonically increases as a function of $R$ and is positive only provided

$$
R>R_{c}=R_{s} / s,
$$

where $R_{s}=2 \gamma \Omega / k_{B} T$ and $s=\ln \Delta$. The case of $R=R_{c}$ corresponds to an equilibrium (the void neither shrinks, no grows), but the equilibrium is an unstable one.

A more complicated situation is met, when the material accumulates during irradiation a certain amount of gas (typically, helium) that is poorly soluble in the matrix and precipitates in the available empty spaces, including vacancies and small cavities (vacancy clusters). When helium is accumulated in a cavity, it prevents complete cavity dissolution. Hence, a gas-containing cavity (gas bubble) should have, in addition to $R_{c}$, one more equilibrium size, $R_{g}$, which answers the situation where the cavity surface tension is approximately counterbalanced by the gas pressure inside the bubble.

The expression for the equilibrium radius $R_{c}$ in the case of gas bubbles is given by a more complicated relation than equation (4.6), because the surface traction in equation (4.4) is now described as

$$
\sigma_{0}=P-\frac{2 \gamma}{R}
$$

where $P$ is the internal gas pressure in the cavity, which is also sensitive to the bubble size. For a bubble containing a fixed number of gas atoms, $n_{g}$, one has to use an appropriate equation of state (EOS) in order to relate pressure to the bubble volume. A typical equation of state can be written down as

$$
P=\xi k_{B} T F\left(\xi V_{g}\right)
$$


where $\xi=n_{g} / V$ is the gas density in the cavity, $V_{g}$ - the gas atom 'volume', and $F$ - some function of the gas density, which tends to unity, when $\xi \rightarrow 0$. In the latter case, the equation of state transforms into the ideal gas equation. Assuming it for simplicity (in spite of the fact that for small gas bubbles the ideal gas approximation works poorly), the equation for the equilibrium bubble radii can be written down in the form

$$
Q\left(R, n_{g}\right)=0
$$

where we have introduced the notation

$$
Q\left(R, n_{g}\right)=\frac{3 n_{g} \Omega}{4 \pi R^{3}}-\frac{R_{s}}{R}+s .
$$

When treated as a function of $R$ at a fixed value of $n_{g}, Q$ has a unique minimum at the void size

$$
R_{\min }=\sqrt{\frac{9 n_{g}}{4 \pi} \frac{\Omega}{R_{s}}},
$$

while the value of function $Q$ in the minimum is

$$
Q\left(R_{\min }\right)=s-\frac{2}{3} \frac{R_{s}}{R_{\min }} .
$$

It is seen that equation (4.9) can be satisfied only provided $Q\left(R_{\min }\right) \leq 0$, or

$$
s \leq \frac{2}{3} \frac{R_{s}}{R_{\min }} .
$$

Otherwise, $Q$ is positive for all $R$, meaning that bubbles of all sizes tend to grow. The limiting case for the existence of positive roots of $Q$ corresponds to exact equality, when

$$
R_{\min }=R^{*}=\frac{2}{3} \frac{R_{s}}{s}
$$

and the number of gas atoms in such cavity, according to eq. (4.11), equals to

$$
n_{g}^{*}=\frac{16 \pi}{81 s^{2} \Omega} R_{s}^{3} \text {. }
$$

It is convenient to use these $R^{*}$ and $n_{g}^{*}$ values in order to introduce non-dimensional variables $\rho=R / R^{*}$ and $\eta_{g}=n_{g} / n_{g}^{*}$ as normalized bubble radius and normalized gas content in the bubble, respectively. In these variables, $Q$ can be expressed in a very simple form,

$$
Q(\rho, \eta)=\frac{3}{2} s\left[\frac{1}{3} \frac{\eta_{g}}{\rho^{3}}-\frac{1}{\rho}+\frac{2}{3}\right]
$$

Finally, when expressed in the dimensionless variables, equations (4.11) and (4.12) are reduced to 


$$
\rho_{\min }=\sqrt{\eta_{g}} \quad \text { and } \quad Q\left(\rho_{\min }\right)=\frac{s}{\rho_{\min }}\left(\rho_{\min }-1\right)
$$

The plot of $Q$ as a function of normalized bubble radius $\rho$ at different values of $\eta_{g}$ is shown in Fig.7.

$<$ Fig7 $>$

As can be seen, $Q\left(\rho, \eta_{g}\right)$ has two roots for $\eta_{g}<1$. The smaller one corresponds to stable equilibrium size $R_{g}$ and the larger one - to the critical size for unlimited cavity growth, $R_{u}$. In practice, when insoluble gas is introduced into the matrix gradually, during either irradiation (e.g. via transmutation reactions) or ion implantation, the growth of cavities initially is only possible due to gas atom accumulation in vacancy clusters. Typically, in irradiation/implantation conditions the rate of vacancy production in metals is much higher than that of helium accumulation and the experimental temperatures are high enough to guarantee vacancy mobility. So, when a cavity captures a gas atom, its volume can accommodate to the new gas content, corresponding to the lower root of $Q$. However, this process continues only while the gas atom number in the cavity remains smaller than $n_{g}^{*}$. When this limit is exceeded, the cavity does not need more gas atoms in order to grow and grows in a void-like mode, not caring about further accumulation of gas atoms (even if it continues). For this reason, $n_{g}^{*}$ is usually referred to as the critical number of gas atoms for the bubble-to-void transition and $R^{*}$ - as the bubble-to-void transition radius.

\subsection{Gas bubble growth on second-phase particles}

Let us consider now a spherical bubble that grows on a spherical second-phase particle and thus has a shape shown in Fig. 2(b). The cavity growth law can still be described by equation (4.2), but the cavity bias factors are now given by more complicated expressions than for a cavity in the bulk. However, as far as there is no substantial difference between $Y_{v}$ and $Y_{i}$, this is not important for the problem we consider. It is also not a priori evident how the expression for the factor $\Delta_{v}^{\text {th }}$ should look like, but it can be easily shown that equation (4.4) remains applicable for a spherical cavity segment on a spherical substrate.

Indeed, when the surface tension can differ on different segments of the cavity surface, $S$, the equilibrium vacancy concentration $C_{v}^{\text {th }}$ at the cavity/matrix interface can be obtained from the general requirement that the system free energy should be at minimum when the cavity is in equilibrium with a solution of vacancies in the environment. The change of the system free energy, $d F$, on adding one vacancy (i.e. a small volume $d V=\Omega$ ) to the cavity can be written down as 


$$
d F=-P d V+d\left(\int_{S} \gamma d S\right)-\mu,
$$

where $\mu$ is the chemical potential (per vacancy) of vacancies in the bulk and integration of surface tension is over the whole cavity surface. Having in mind that the average vacancy concentration in the bulk is extremely low even in the ion implantation conditions (simple estimates indicate that in our experimental conditions it does not exceed $10^{-9}$, one can use for $\mu$ the dilute solution approximation, namely [48]

$$
\mu=E_{v}^{f}+k_{B} T \ln C_{v} .
$$

In equilibrium one has $d F=0$, which immediately gives for the equilibrium concentration the relation

$$
C_{v}^{t h}=C_{v 0}^{t h} \exp \left(-\frac{\Omega}{k_{B} T}\left(P-\frac{d}{d V}\left(\int_{S_{c}} \gamma d S\right)\right)\right) .
$$

Since we assume that the cavity shape at a small change of the cavity volume quickly accommodates itself to the equilibrium one, being always represented by two spherical interfaces (one with the substrate and another with the matrix), then

$$
\Delta_{v}^{t h} \equiv \exp \left(-\left[P-\gamma \frac{d S_{c}}{d V_{c}}-\left(\gamma_{s c}-\gamma_{s m}\right) \frac{d S_{s c}}{d V_{c}}\right] \frac{\Omega}{k_{B} T}\right),
$$

where $V_{c}$ it the volume of the cavity, $S_{c}$ - the area of the cavity-matrix surface, and $S_{s c}$ - the area of the cavity-substrate interface. Taking into account equation (3.3), this is reduced to

$$
\Delta_{v}^{t h} \equiv \exp \left(-\left[P-\gamma \frac{d \Delta S_{c}}{d V_{c}}\right] \frac{\Omega}{k_{B} T}\right),
$$

where $\Delta S_{c}=S_{c}-S_{s c} \cos \alpha$.

In order to calculate the derivative $d \Delta S_{c} / d V_{c}$, one needs explicit equations for the volume and surface area of a cavity that lies on a spherical particle with radius $R_{p}$. The volume is given by equation (3.2), while the segment surface areas are

$$
S_{c}=2 \pi R^{2}(1-\cos (\alpha+\beta)) \quad \text { and } \quad S_{s c}=2 \pi R_{p}^{2}(1-\cos \beta),
$$

where angles $\alpha$ and $\beta$ are as defined in Fig. 2(b). Having in mind equation (3.4), the cosines in equation (4.22) can be written down in a compact form as

$$
\cos (\alpha+\beta)=\frac{a}{\mathfrak{R}} \quad \text { and } \quad \cos \beta=\frac{b}{\mathfrak{R}},
$$

where we have introduced new variables $a=\cos \alpha-\zeta, b=1-\zeta \cos \alpha, \Re=\sqrt{1-2 \zeta \cos \alpha+\zeta^{2}}$, and $\zeta=R / R_{p}$. With these variables, one can write 


$$
\Delta S_{c}=S_{c}-\cos \alpha S_{s c}=2 \pi R_{p}^{2}\left[\zeta^{2}\left(1-\frac{a}{\mathfrak{R}}\right)-\cos \alpha\left(1-\frac{b}{\mathfrak{R}}\right)\right]
$$

and

$$
V_{c}=\frac{\pi}{3} R_{p}^{3}\left[\zeta^{3}\left(2-3 \frac{a}{\mathfrak{R}}+\frac{a^{3}}{\mathfrak{R}^{3}}\right)-\left(2-3 \frac{b}{\mathfrak{R}}+\frac{b^{3}}{\mathfrak{R}^{3}}\right)\right],
$$

so that the derivative $d \Delta S_{c} / d V_{c}$, can be calculated as

$$
\frac{d \Delta S_{c}}{d V_{c}}=\frac{d \Delta S_{c}}{d \zeta} / \frac{d V_{c}}{d \zeta}
$$

The derivatives over $\zeta$ are obtained straightforwardly,

$$
\frac{d \Delta S_{c}}{d \zeta}=2 \pi R_{p} R\left(2-2 \frac{a}{\mathfrak{R}}-\frac{a \sin ^{2} \alpha}{\mathfrak{R}^{3}}\right) \quad \text { and } \quad \frac{d V_{c}}{d \zeta}=\pi R_{p} R^{2}\left(2-2 \frac{a}{\mathfrak{R}}-\frac{a \sin ^{2} \alpha}{\mathfrak{R}^{3}}\right)
$$

and hence

$$
\frac{d \Delta S_{c}}{d V_{c}}=\frac{2}{R}
$$

which has exactly the same form as for a cavity in the bulk. Thus, equation (4.21) is reduced to equation (4.4).

The equation for the equilibrium bubble radii can then be written down in the form identical to equation (4.9), where function $Q$ has the from (assuming the ideal gas law for the gas pressure in the cavity),

$$
Q\left(R, n_{g}\right)=\frac{n_{g} \Omega}{V_{c}}-\frac{R_{s}}{R}+s .
$$

The only difference of equation (4.26) from equation (4.10) is that the volume of the cavity is now smaller than that for the cavity in the bulk.

In a special case considered in Ref. [17], where cavity lies on a flat matrix-substrate interface $(\beta=0)$, the equation for the equilibrium bubble radii can be written down in terms of non-dimensional void radius and gas content as

$$
\left.Q\left(R, n_{g}\right)=\frac{3 s}{2}\left[\frac{4}{3(2+\cos \alpha)(1-\cos \alpha)^{2}} \frac{\eta_{g}}{\rho^{3}}-\frac{1}{\rho}+\frac{2}{3}\right)\right]=0 .
$$

The minimum of $Q$ lies at the relative void size $\rho_{\min }$, defined by equation

$$
\rho_{\min }=\sqrt{\frac{4}{\left.(1-\cos \alpha)\left[2-\cos \alpha-\cos ^{2} \alpha\right)\right]} \eta_{g}},
$$

while the function value at $\rho=\rho_{\min }$ is 


$$
Q\left(\rho_{\min }, n_{g}\right)=\frac{s}{\rho_{\min }}\left[\rho_{\min }-1\right]
$$

The bubble-to-void transition occurs when $Q\left(\rho_{\min }\right)=0$, that is at $\rho_{\min }=1$, exactly the same as for the bubble in the bulk. However, the critical number of gas atoms required to reach the transition radius differs, being equal to

$$
n_{g l}^{*}=\frac{(1-\cos \alpha)^{2}[2+\cos \alpha]}{4} n_{g}^{*} \equiv f_{l} n_{g}^{*}
$$

Depending on $\alpha$, the factor $f(\alpha)$ varies as shown in Fig. 8, never exceeding unity. So in this particular limiting case the bubble-to-void transition radius $R^{*}$ for a spherical bubble segment does not change as compared to the bubble in the bulk, but the critical number of gas atoms decreases for all wetting angles $\alpha$.

$<$ Fig8 $>$

Now let us consider a cavity located on a spherical particle with radius $R_{p}$. As can be noticed in equation (4.24), the cavity volume in this case is a function of the ratio $\zeta=R / R_{p}$. The plot of normalized cavity volume $v_{c}=3 V_{c} /\left(4 \pi R^{3}\right)$ as a function of $\zeta$ is shown in Fig.9 for different values of wetting angle $\alpha$.

$<$ Fig9 $>$

In non-dimensional variables $\rho$ and $\eta_{g}$, the equation for equilibrium cavity radii is reduced to

$$
\left.Q\left(R, n_{g}\right)=\frac{3 s}{2}\left[\frac{1}{3 v_{c}(\rho)} \frac{\eta_{g}}{\rho^{3}}-\frac{1}{\rho}+\frac{2}{3}\right)\right]=0 .
$$

The location of the minimum of this function can be found from equation

$$
\eta_{g}=v_{c}\left(\rho_{\min }\right)\left(1-2 \chi\left(\zeta_{p}^{*} \rho_{\min }, \alpha\right)\right) \rho_{\min }^{2},
$$

while the value of the function in the minimum point is

$$
Q\left(\rho_{\min }\right)=\frac{s}{\rho_{\min }}\left[\rho_{\min }-1-\chi\left(\zeta_{p}^{*} \rho_{\min }, \alpha\right)\right]
$$

where $\zeta_{p}^{*}=R^{*} / R_{p}$ and

$$
\chi(\zeta, \alpha)=\frac{(2+\cos \beta)(1-\cos \beta)^{2}}{2 \zeta^{3}(2+\cos (\alpha+\beta))(1-\cos (\alpha+\beta))^{2}} .
$$

It is worth mentioning that the minimum value of $Q$ in the ideal gas approximation is insensitive to the number of gas atoms in the bubble. 
The dependence of $\chi$ on the void to particle radii ratio $\zeta$ is shown in Fig. 10 for different values of the wetting angle. In the limiting cases of small and large $\zeta$ it is described, respectively, by the relations

$$
\chi(\zeta, \alpha) \approx \frac{3}{8} \frac{(1+\cos \alpha)^{2}}{(2+\cos \alpha)} \zeta \quad \text { and } \quad \chi(\zeta, \alpha) \approx \frac{(2-\cos \alpha)(1+\cos \alpha)^{2}}{8 \zeta^{3}}
$$

$<$ Fig $10>$

An implicit equation for the bubble-to-void transition radius $\rho_{p}^{*}$ is obtained by equating $Q\left(\rho_{p}^{*}\right)$ to zero, which gives

$$
\rho_{p}^{*}=1+\chi\left(\zeta_{p}^{*} \rho_{p}^{*}, \alpha\right)
$$

Since $\chi$ is a positive function for all possible arguments, the bubble-to-void transition radius for a cavity on a spherical particle is larger than for similar size bubbles in the bulk or on a flat substrate. But the increase of $\rho_{p}^{*}$ is relatively small for all particle sizes, especially when the wetting angle is not too close to zero. In a zero-order approximation we can get an analytical parametric dependence of $\rho_{p}^{*}$ on the relative bubble size by setting $\rho_{p}^{*}=1$ in the r.h.s. of equation (4.35),

$$
\rho_{p a}^{*}=1+\tilde{\chi}\left(\zeta_{p}^{*}, \alpha\right)
$$

The difference between the exact values of $\rho_{p}^{*}$ and the approximate ones obtained using this simplification is shown in Fig. 11(a).

$<$ Fig11 $>$

Substituting $\rho_{p}^{*}$ into equation (4.33), one obtains the equation for the critical number of gas atoms, $\eta_{g p}^{*}$, required to reach the bubble-to-void transition size,

$$
\eta_{g p}^{*}=v_{c}\left(\zeta_{p}^{*} \rho_{p}^{*}, \alpha\right)\left(1-2 \chi\left(\zeta_{p}^{*} \rho_{p}^{*}, \alpha\right)\right) \rho_{p}^{* 2} .
$$

The dependence of $\eta_{g p}^{*}$ on the normalized particle size $R_{p} / R^{*}$ is shown in Fig. 11(b). The same figure shows also the approximate analytical dependences obtained by setting $\rho_{p}^{*}=1$ in the first two terms in the r.h.s. of equation (4.37), namely

$$
\eta_{g p}^{*} \approx v_{c}\left(\zeta_{p}^{*}, \alpha\right)\left(1-2 \chi\left(\zeta_{p}^{*}, \alpha\right)\right) \rho_{p a}^{* 2} .
$$

It can be seen that eq. (4.38) gives a reasonable approximation of the critical gas content in the bubble for wetting angles exceeding roughly $45^{\circ}$. 
Finally, let us discuss how the predictions for the critical bubble size and gas content will change if we discard the ideal gas approximation. In this case the relation between the gas pressure and the gas density in the bubble is given by relation (4.8). Correspondingly, the equation determining the equilibrium bubble radii has the form

$$
\left.Q\left(R, n_{g}\right)=\frac{3 s}{2}\left[\frac{1}{3 v_{c}(\rho)} \frac{\eta_{g}}{\rho^{3}} F\left(\theta \frac{\eta_{g}}{v_{c}(\rho) \rho^{3}}\right)-\frac{1}{\rho}+\frac{2}{3}\right)\right]=0,
$$

which can be rewritten in an equivalent form as

$$
n_{g} z F\left(\theta n_{g} z\right)=\left(\frac{3}{\rho}-2\right)
$$

where

$$
\theta=\frac{s V_{g}}{2 \Omega}
$$

and

$$
z=\frac{1}{\rho^{3} v_{c}(\rho)}
$$

The value $\rho_{\min }$ of cavity radius in the minimum of function $Q$ is determined by the requirement of vanishing derivative of $Q$ at $\rho_{\mathrm{min}}$, which is equivalent to

$$
z \frac{d}{d z}\left[\eta_{g} z F\left(\theta \eta_{g} z\right)\right]_{z=z\left(\rho_{\min }\right)}=\frac{\left(1-2 \chi\left(\zeta_{p} \rho_{\min }, \alpha\right)\right.}{\rho_{\min }} .
$$

Using now the fact that for a critical bubble both equations (4.40) and (4.43) should be satisfied simultaneously, the equations for the critical gas atom number $\eta_{g p}^{*}$ and critical radius $\rho_{p}^{*}$ can be written down in the form closely resembling equations (4.32) and (4.35),

$$
\eta_{g p}^{*}=\frac{F_{1}\left(\theta \eta_{g p}^{*} z\left(\rho_{p}^{*}\right)\right)}{F\left(\theta \eta_{g p}^{*} z\left(\rho_{p}^{*}\right)\right)}\left(1-2 \chi\left(\zeta_{p} \rho_{p}^{*}, \alpha\right)\right) \rho_{p}^{* 3} v_{c}\left(\rho_{p}^{*}\right)
$$

and

$$
\rho_{p}^{*}=\frac{3}{2}-\frac{1}{2} F_{1}\left(\theta \eta_{g p}^{*} z\left(\rho_{p}^{*}\right)\right)\left[1-2 \chi\left(\zeta_{p} \rho_{p}^{*}, \alpha\right)\right]
$$

where

$$
F_{1}(y)=\frac{1}{\left[1+y \frac{d}{d y}[\ln F(y)]\right]} .
$$

These relations lead to some general conclusions even without specifying a particular form of EOS. First of all, whatever is the equation of state, one has $F(0)=F_{1}(0)=1$. Second, when the argument $y$ (proportional to the number of gas atoms in the cavity) increases, the function $F(y)$ 
grows monotonically, tending to infinity as $y \rightarrow 1$. Correspondingly, $F_{1}(y \rightarrow 1) \rightarrow 0$. Thus, both $F_{1}(y)$ and $F_{1} / F$ vary within the interval $[0,1]$. Since $1-2 \chi$ also varies within the same limits (see Fig. 10), the account of EOS deviation from the ideal gas law results in the shift of critical bubbleto-void radius from 1 closer to 1.5 . For the critical number of gas atoms the trend is less clear and requires knowledge of the particular equation of state.

Let us consider, for example, hard-sphere equation of state (HSEOS) in the CarnahanStarling approximation [49], which has the form of equation (4.8) with

$$
F(y)=\frac{1+y+y^{2}-y^{3}}{(1-y)^{3}} .
$$

In the case when the cavity filling gas is helium, the gas atom 'volume' $V_{g}$ can be taken equal to $5.42 \AA^{3}$ [50]. Equations (4.44), (4.45) become now coupled and allow only numerical solution that depends on three parameters, namely $\alpha, R_{p}$, and $\theta$. The latter is defined in equation (4.41) and is directly proportional to the efficient vacancy supersaturation, $\theta \cong 0.23 \mathrm{~s}$, where we have used the above-mentioned value of $V_{g}$ and $\Omega=11.8 \AA^{3}$ for the atomic volume of iron. The calculated plots of $\eta_{g p}^{*}$ and $\rho_{p}^{*}$ as functions of the particle radius are shown in Fig. 12 for different values of $s$ and $\alpha$.

\section{$<$ Fig12>}

As can be seen, the normalized critical radii are predicted to be always larger than the corresponding values for the ideal gas approximation, while the normalized gas density in the bubble is lower. It is worth mentioning that the trend remains valid in the limiting case $\alpha \rightarrow 180^{\circ}$, when the solution becomes formally equivalent to that for a spherical gas bubble in the bulk. The latter case was considered earlier in Ref. [7], where, having in mind the corrected presentation of calculated results given later in Ref. [42], exactly the same trend was predicted.

\section{Discussion}

As follows from the theoretical considerations in sect. 4, helium bubbles that grow on oxide particles require less gas atoms for the bubble-to-void transition as compared to bubbles in the bulk, in spite of slightly larger critical radius. Hence it is quite reasonable that the oxide particles serve as triggers for the bubble-to void-transition. It is interesting to estimate, however, how good the model can perform in describing the effect of $\mathrm{dpa} / \mathrm{He}$ ratio increase on the onset of bubble-tovoid transition in quantitative terms.

According to equation (4.14), the critical radius $R^{*}$ for the bubble-to-void transition is inversely proportional to the efficient vacancy supersaturation, which relates, in turn, to the average point defect concentrations in the matrix that are determined by the balance of point defect 
generation rate by irradiation, $G$, and the strengths of internal sinks for point defects, $k_{v}^{2}$ and $k_{i}^{2}$, as

$$
D_{m} C_{m}=\frac{G}{k_{m}^{2}}
$$

Using these relations, one can re-write $s$ as

$$
s=\ln \left(\frac{k_{i}^{2}-k_{v}^{2}}{k_{i}^{2} k_{v}^{2}} \frac{G}{D_{v} C_{v 0}^{t h}}\right),
$$

The sink strengths in this equation are total sink strengths that are calculated as the sums of strengths of all point defect sinks available in the material [51],

$$
k_{m}^{2}=\sum_{j} k_{m j}^{2}
$$

where $j$ runs over all possible sinks. Four major groups of sinks are present in ODS-Eurofer steel, namely dislocations, grain boundaries, oxide particles and cavities, some of which are located in the bulk and some are attached to other structural defects. When estimating the sink strengths, we assume that the presence of cavities on dislocations and grain boundaries does not affect much the sink strengths of extended defects and estimate them according to standard relations. In particular, for dislocations we assume [51]:

$$
k_{m d}^{2}=Z_{m} \rho_{d},
$$

where $\rho_{d}$ is the dislocation density and $Z_{m}$ - the dislocation bias factors, determined as

$$
Z_{m}=\frac{4 \pi}{\ln \left(1 / \pi \rho_{d} R_{d m}^{2}\right)},
$$

where $R_{d m}$ are the dislocation capture radii for point defects of type $m$. For numerical estimates the latter can be taken equal to $R_{d i}=10 b$ and $R_{d v}=4 b$, where $b$ is the dislocation Burgers vector [52].

The grain boundary sink strength can be estimated as [53]

$$
k_{m g}^{2}=\frac{15}{\left(d_{g} / 2\right)^{2}},
$$

where $d_{g}$ is the effective grain diameter, which was estimated for the elongated grains of ODSEurofer steel as the average of the mean grain length and width. Sink strength of He-filled cavities in the bulk is calculated as [51]

$$
k_{m c}^{2}=4 \pi r_{c} N_{c}
$$


where $r_{c}$ and $N_{c}$ are the average cavity radius and number density in the grain bulk. Finally, having in mind that all $\mathrm{Y}_{2} \mathrm{O}_{3}$ particles are covered with single cavities with the size comparable to that of the particle, the sink strength of such particle-bubble complexes can be roughly estimated as

$$
k_{m p}^{2}=4 \pi r_{c}^{e f f} N_{p}
$$

where $r_{c}^{e f f}=\sqrt{\left(r_{c}^{p}\right)^{2}+\left(r_{p}\right)^{2}}$ is the effective radius of the oxide-cavity complex, $r_{p}$ and $r_{c}^{p}$ are the average radii of oxide nanoparticles and the associated cavities, respectively, and $N_{p}$ is the particle number density.

Most of the input parameters necessary for the estimation of sink strengths can be found in Tables 1 and 2, while the resulting sink strengths are collected in Table 3 for both irradiation regimes used in the current study. Taking the self-diffusion coefficient equal to $D_{v} C_{v 0}^{\text {th }}=2.76 \exp \left(-3.0 \mathrm{eV} / k_{B} T\right) \mathrm{cm}^{2} / \mathrm{c}$ [54] and assuming the temperature of $823 \mathrm{~K}$, we get $s \cong 1.7$ for the single-beam helium implantation case $\left(G=4 \times 10^{-5} \mathrm{dpa} / \mathrm{s}\right)$ and $s \cong 5.9$ for the dual-beam irradiation $\left(G=2.4 \times 10^{-3} \mathrm{dpa} / \mathrm{s}\right)$. In other words, the critical radius of bubble-to-void transition in the dual-beam irradiation case should be more than three times lower than in the case of singlebeam implantation, even if we take into account the correction due to the non-ideal helium EOS in the bubbles. Qualitatively, this can explain the experimental observation that the bubble-to-void transition has occurred only in the dual-beam irradiated sample.

At the same time, the critical radii predicted by the theory are definitely too small. For the typical values $\gamma=2 \mathrm{~J} / \mathrm{m}^{2}$ and $\Omega=11.8 \AA^{3}$, we get $R_{c}=4.2 \mathrm{~nm}$, which would mean the critical cavity diameter of $3.3 \mathrm{~nm}$ for single-beam irradiation, while for the dual-beam case it would be only $\sim 1 \mathrm{~nm}$. The account of the non-ideal helium behaviour in the bubbles increases these estimates, but no more than by 20-30\%. In reality it should be at least an order of magnitude larger because, judging from Fig. 5, the transition starts from the bubble diameter $\sim 10 \mathrm{~nm}$. It should be kept in mind, however, that a discrepancy in quantitative estimates of critical cavity size is quite common in the literature and can be due, in particular, to the neglect of the cavity size dependence of cavity bias factors, which is non-negligible for cavities in the nanometer size range [47].

Finally, it is worth mentioning that, as can be noticed in Fig. 11(b), small particles (with $\left.R_{p} / R^{*}<1\right)$ weakly affect the critical gas content $\eta_{g p}^{*}$ in particle-associated cavities, whatever is the wetting angle. Only particles with the size comparable or larger than the critical one in the bulk remarkably decrease the critical gas content in the bubble. In practical terms, this means that only sufficiently large second phase particles can be efficient triggers for the bubble-to-void transition, promoting the void swelling onset. This prediction is in agreement with both the current 
experimental results and the earlier findings $[18,19,22]$. The effect is especially pronounced for moderate wetting angles, including that typical for cavities on yttria particles.

\section{Summary}

The most important findings of this study can be summarized as follows.

(1) The qualitative patterns of bubble formation in ODS-Eurofer steel implanted at $823 \mathrm{~K}$ with $10 \mathrm{keV} \mathrm{He}$ ions to $\sim 8.5 \times 10^{3}$ appm He in either a single beam regime or simultaneously with a secondary $4 \mathrm{MeV} \mathrm{Au}^{2+}$ ion beam are largely similar in spite of a strong increase of radiation damage production by the secondary beam. In both cases, one observes the partitioning of bubbles between the bulk and various microstructural defects - dislocations, grain boundaries and oxide nanoparticles. In quantitative terms, the effect of the damage generation rate increase is equivalent to the effective temperature shift towards higher temperatures, being manifested in the decrease of bubble number density and the increase of bubble average size for all bubble families. The only exception is the number density of bubbles on oxide nanoparticles, which remains in one-to-one correspondence with the particle number density.

(2) The only important qualitative effect caused by strong acceleration of damage production with a secondary gold beam is the formation of large cavities on some oxide particles. The effect is interpreted as a manifestation of the bubble-to-void transition caused by the increase of the effective vacancy supersaturation in the dual-beam irradiated sample.

(3) The estimated swelling due to visible He bubbles is increased by the dual-beam $\mathrm{He}^{+}+$ $\mathrm{Au}^{2+}$ irradiation as compared to single-beam $\mathrm{He}^{+}$implantation, but, disregarding the contribution from large voids on oxide particles, the increase is quite moderate and can be rationalized in terms of the effective temperature shift associated with the increased damage production by the gold ion beam. However, the bubble-to-void transition, even though taking place on a minor part of oxide particles, bears potential risk of strongly accelerating swelling.

(4) A theoretical model of bubble-to-void transition for gas bubbles on second-phase particles is developed. The model predicts that the critical radius for bubble-to-void transition for such bubbles increases (up to $50 \%$, depending on the bubble and particle parameters) as compared to that for bubbles in the bulk, but the critical number of gas atoms promoting the transition decreases. The effect is most pronounced for relatively large host particles, which are thus the most probable candidates to launch the bubble-to-void transition, which agrees well with the available experimental observations. 


\section{Acknowledgements}

The authors acknowledge the efforts of the JANNuS-Orsay/SCALP technical staff of the IJCLab, especially Sandrine Picard, Jérome Bourçois and Cédric Baumier. O.Emelyanova is deeply grateful to Vernadskiy program of French embassy in Moscow and Université Paris-Sud for financial support of the joint Ph.D. thesis execution.

Computational part of this work was carried out using high-performance computing resources of federal center for collective usage at NRC "Kurchatov Institute", http://computing.kiae.ru/.

The work has received funding from the Governmental Support of Competitive Growth Program of NRNU MEPhI (agreement No. 02.a03.21.0005).

The work has been carried out within the framework of the French Research Federation for Fusion Studies and EUROfusion consortium, and has received funding from the Euratom research and training programme 2014-2018 and 2019-2020 under agreement No. 633053. The views and opinions expressed herein do not necessarily reflect those of the European Commission.

\section{Appendix. Derivation of equations (3.4)}

Equations (3.4) can be easily derived if one notices that the intersection line of two spheres (a particle and a void) is a circle, whose radius $\mathrm{r}$ can be expressed as either $r_{0}=R_{p} \sin \beta$ or $r_{0}=R \sin (\alpha+\beta)$, see Fig. 2(b). Hence

$$
R_{p} \sin \beta=R \sin (\alpha+\beta)=R(\sin \alpha \cos \beta+\sin \beta \cos \alpha),
$$

which is equivalent to

$$
\tan \beta=\frac{R \sin \alpha}{\left(R_{p}-R \cos \alpha\right)} .
$$

This immediately gives the first of two formulae in equation (3.4),

$$
\cos \beta=\sqrt{\frac{1}{1+\tan ^{2} \beta}}= \pm \frac{R_{p}-R \cos \alpha}{\sqrt{R_{p}^{2}-2 R R_{p} \cos \alpha+R^{2}}},
$$

where positive sign should be retained when angle $\beta$ is defined as in Fig. 2(b). The second one is obtained combining equations (A.1) and (A.2) as

$$
\cos (\alpha+\beta)=\sqrt{1-\frac{R_{p}^{2}}{R^{2}}\left(1-\cos ^{2} \beta\right)}=\frac{R_{p} \cos \alpha-R}{\sqrt{R_{p}^{2}-2 R R_{p} \cos \alpha+R^{2}}} .
$$




\section{Data availability}

The raw/processed data required to reproduce these findings cannot be shared at this time as the data also forms part of an ongoing study.

\section{Declaration of competing interest}

No competing interests to declare.

\section{References}

[1] P. Vladimirov, A. Möslang, Comparison of material irradiation conditions for fusion, spallation, stripping and fission neutron sources, J. Nucl. Mater. 329-333 (2004) 233-237. https://doi.org/10.1016/j.jnucmat.2004.04.030

[2] R. Lindau, M. A, M. Schirra, P. Schlossmacher, M. Klimenkov, Mechanical and microstructural properties of a hipped RAFM ODS-steel, J. Nucl. Mater. 307-311. 307-311 (2002) 769-772. https://doi.org/10.1016/S0022-3115(02)01045-0

[3] S.J. Zinkle, J.L. Boutard, D.T. Hoelzer, A. Kimura, R. Lindau, G.R. Odette, M. Rieth, L. Tan, H. Tanigawa, Development of next generation tempered and ODS reduced activation ferritic/martensitic steels for fusion energy applications, Nucl. Fusion. 57 (2017) 092005. https://doi.org/10.1088/1741-4326/57/9/092005.

[4] G.R. Odette, N.J. Cunningham, T. Stan, M.E. Alam, Y. De Carlan, Nano-Oxide DispersionStrengthened Steels, in: R. Odette, S. Zinkle (Eds.),Structural Alloys for Nuclear Energy Applications, Elsevier, Amsterdam, 2019, pp. 529-583. https://doi.org/10.1016/B978-0-12$\underline{397046-6.00012-5}$

[5] G.R. Odette, D.T. Hoelzer, Irradiation-tolerant nanostructured ferritic alloys: Transforming helium from a liability to an asset, JOM 62 (2010) 84-92. https://doi.org/10.1007/s11837-010$\underline{0144-1}$

[6] F.A. Garner, M.B. Toloczko, B.H. Sencer, Comparison of swelling and irradiation creep behavior of fcc-austenitic and bcc-ferritic/martensitic alloys at high neutron exposure, J. Nucl. Mater. 276 (2000) 123-142. https://doi.org/10.1016/S0022-3115(99)00225-1.

[7] R.E. Stoller, G.R. Odette, Analytical solutions for helium bubble and critical radius parameters using a hard sphere equation of state, J. Nucl. Mater. 131 (1985) 118-125. https://doi.org/10.1016/0022-3115(85)90450-7

[8] L.K. Mansur, W.A. Coghlan, Mecanisms of helium interaction with radiation effects in metals and alloys: a review, J. Nucl. Mater. 119. 119 (1983) 1-25. https://doi.org/10.1016/00223115(83)90047-8 
[9] R. Schaeublin, T. Leguey, P. Spaetig, N. Baluc, M. Victoria, Microstructure and mechanical properties of two ODS ferritic / martensitic steels, J. Nucl. Mater. 307-311 (2002) 778-782. https://doi.org/10.1016/S0022-3115(02)01193-5

[10] H.R.Z. Sandim, R.A. Renzetti, A.F. Padilha, D. Raabe, M. Klimenkov, R. Lindau, A. Möslang, Annealing behavior of ferritic-martensitic 9\%Cr-ODS-Eurofer steel, Mater. Sci. Eng. A. 527 (2010) 3602-3608. https://doi.org/10.1016/j.msea.2010.02.051

[11]R. Lindau, M. Klimenkov, U. Jäntsch, A. Möslang, L. Commin, Mechanical and microstructural characterization of electron beam welded reduced activation oxide dispersion strengthened - Eurofer steel, J. Nucl. Mater. 416 (2011) 22-29.

https://doi.org/10.1016/j.jnucmat.2011.01.025

[12] D. Brimbal, L. Beck, O. Troeber, E. Gaganidze, P. Trocellier, J. Aktaa, R. Lindau, Microstructural characterization of Eurofer-97 and Eurofer-ODS steels before and after multibeam ion irradiations at JANNUS Saclay facility, J. Nucl. Mater. 465 (2015) 236-244. https://doi.org/10.1016/j.jnucmat.2015.05.045

[13] S. Rogozhkin, A. Bogachev, O. Korchuganova, A. Nikitin, N. Orlov, A. Aleev, A. Zaluzhnyi, M. Kozodaev, T. Kulevoy, B. Chalykh, R. Lindau, J. Hoffmann, A. Möslang, P. Vladimirov, M. Klimenkov, M. Heilmaier, J. Wagner, S. Seils, Nanostructure evolution in ODS steels under ion irradiation, Nucl. Mater. Energy. 9 (2016) 66-74.

https://doi.org/10.1016/j.nme.2016.06.011

[14] C.M. Parish, K.A. Unocic, L. Tan, S.J. Zinkle, S. Kondo, L.L. Snead, D.T. Hoelzer, Y. Katoh, Helium sequestration at nanoparticle-matrix interfaces in helium $\mathrm{p}$ heavy ion irradiated nanostructured ferritic alloys *, J. Nucl. Mater. 483 (2017) 21-34.

https://doi.org/10.1016/j.jnucmat.2016.10.038

[15] P.D. Edmondson, C.M. Parish, Y. Zhang, A. Hallén, M.K. Miller, Helium bubble distributions in a nanostructured ferritic alloy, J. Nucl. Mater. 434 (2013) 210-216. https://doi.org/10.1016/j.jnucmat.2012.11.049

[16] M.K. Miller, P.D. Edmondson, C.M. Parish, Y. Zhang, A. Halle, Helium entrapment in a nanostructured ferritic alloy, Scr. Mater. 65 (2011) 731-734.

https://doi.org/10.1016/j.scriptamat.2011.07.024

[17] R.E. Stoller, G.R. Odette, The effect of helium on swelling in stainless steel: influence of cavity density and morphology, in: H. Brager, J. Perrin (Eds.), Effects of Radiation on Materials: Eleventh Int. Symp., ASTM International, West Conshohocken, 1982, pp. 275-294.

https://doi.org/10.1520/STP34351S 
[18] B. Yao, D.J. Edwards, R.J. Kurtz, G.R. Odette, T. Yamamoto, Microstructure Characterization of Neutron Irradiated and Helium Injected PM2000, 14YW, and Modified F82H Alloys, Fusion Mater. Semiannul Prog. Rep. June 2012. DOE-ER-031 (2012) 26. [19] H.J. Jung, D.J. Edwards, R.J. Kurtz, G.R. Odette, Y. Wu, T. Yamamoto, Microstructural Summary of ODS Ferritic Alloys (14YW, 14YWT, 12YWT, MA957FR, PM2000) and RAFM Steels (F82H Mod.3-CW, Eurofer97) from JP27 In-Situ He Injection (ISHI) Experiment at 500ㄷ, Fusion Mater. Semiannul Prog. Rep. June 2015. DOE-ER-031 (2015) 55.

[20] L. Horton, L. Mansur, Experimental Determination of the Critical Cavity Radius in Fe-10Cr for Ion Irradiation, in: F. Garner and J. Perrin (eds.), Effects of Radiation on Materials: Twelfth Int. Symp., West Conshohocken, ASTM International, 1985, 344-362. https://doi.org/10.1520/STP37372S

[21] K. Asano, Y. Kohno, A. Kohyama, Microstructural evolution of HT9 under dual-beam charged particle irradiation, J. Nucl. Mater. 155-157 (1988) 912-915. https://doi.org/10.1016/0022-3115(88)90440-0

[22] L.L. Hsiung, M.J. Fluss, S.J. Tumey, B.W. Choi, Y. Serruys, F. Willaime, Formation mechanism and the role of nanoparticles in Fe-Cr ODS steels developed for radiation tolerance, Phys. Rev. B. 82 (2010) 184103. https://doi.org/10.1103/PhysRevB.82.184103

[23] S. Taller, Z. Jiao, K. Field, G.S. Was, Emulation of fast reactor irradiated T91 using dual ion beam, J. Nucl. Mater. 527 (2019) 151831. https://doi.org/10.1016/j.jnucmat.2019.151831 [24] C. Zheng, E.R. Reese, K.G. Field, T. Liu, E.A. Marquis, S.A. Maloy, D. Kaoumi, Microstructure response of ferritic/martensitic steel HT9 after neutron irradiation : Effect of temperature *, J. Nucl. Mater. 528 (2020) 151845.

https://doi.org/10.1016/j.jnucmat.2019.151845

[25] A. Gentils, C. Cabet, Investigating radiation damage in nuclear energy materials using JANNuS multiple ion beams, Nucl. Instruments Methods Phys. Res. Sect. B Beam Interact. with Mater. Atoms. 447 (2019) 107-112. https://doi.org/10.1016/j.nimb.2019.03.039

[26] M.P. Short, D.R. Gaston, M. Jin, L. Shao, F.A. Garner, Modeling injected interstitial effects on void swelling in self-ion irradiation experiments, J. Nucl. Mater. 471 (2016) 200-207. https://doi.org/10.1016/j.jnucmat.2015.10.002

[27] L. Shao, C.C. Wei, J. Gigax, A. Aitkaliyeva, D. Chen, B.H. Sencer, F.A. Garner, Effect of defect imbalance on void swelling distributions produced in pure iron irradiated with $3.5 \mathrm{MeV}$ self-ions, J. Nucl. Mater. 453 (2014) 176-181. https://doi.org/10.1016/j.jnucmat.2014.06.002 [28] J.F. Ziegler, M.D. Ziegler, J.P. Biersack, SRIM - The stopping and range of ions in matter (2010), Nucl. Instruments Methods Phys. Res. B. 268 (2010) 1818-1823. 
[29] M. Kirk, X. Yi, M. Jenkins, Characterization of irradiation defect structures and densities by transmission electron microscopy, J. Mater. Res. 30 (2015) 1195-1201.

https://doi.org/10.1557/jmr.2015.19

[30] C.M. Parish, M.K. Miller, Aberration-corrected X-ray spectrum imaging and fresnel contrast to differentiate nanoclusters and cavities in helium-irradiated alloy 14YWT, Microsc. Microanal. 20 (2014) 613-626. https://doi.org/10.1017/S1431927614000312

[31] T. Malis, S.C. Cheng, R.F. Egerton, EELS log-ratio technique for specimen-thickness measurement in the TEM, J. Electron Microsc. Tech. 8 (1988) 193-200.

https://doi.org/10.1002/jemt.1060080206

[32] K. Iakoubovskii, K. Mitsuishi, Y. Nakayama, K. Furuya, Thickness measurements with electron energy loss spectroscopy, Microsc. Res. Tech. 71 (2008) 626-631.

https://doi.org/10.1002/jemt.20597

[33] ASTM E521-16, Standard Practice for Investigating the Effects of Neutron Radiation Damage Using Charged-Particle Irradiation, ASTM International, West Conshohocken, 2016. https://doi.org/10.1520/E0521-16

[34] A.I. Ryazanov, O.K. Chugunov, S.M. Ivanov, S.T. Latushkin, R. Lindau, A. Möslang, A.A. Nikitina, K.E. Prikhodko, E. V. Semenov, V.N. Unezhev, P. V. Vladimirov, Tensile properties and microstructure of helium implanted EUROFER-ODS, J. Nucl. Mater. 442 (2013) S153S157. https://doi.org/10.1016/j.jnucmat.2013.03.080.

[35] R. Raj, M.F. Ashby, Intergranular fracture at elevated temperature, Acta Metall. 23 (1975) 653-666. https://doi.org/10.1016/0001-6160(75)90047-4

[36] C.C. Eiselt, M. Klimenkov, R. Lindau, A. Möslang, H.R.Z. Sandim, A.F. Padilha, D. Raabe, High-resolution transmission electron microscopy and electron backscatter diffraction in nanoscaled ferritic and ferritic-martensitic oxide dispersion strengthened-steels, J. Nucl. Mater. 385 (2009) 231-235. https://doi.org/10.1016/j.jnucmat.2008.11.029.

[37] B. Mazumder, M.E. Bannister, F.W. Meyer, M.K. Miller, C.M. Parish, P.D. Edmondson, Helium trapping in carbide precipitates in a tempered F82H ferritic - martensitic steel, NME. 1 (2015) 8-12. https://doi.org/10.1016/j.nme.2014.11.001.

[38] W. Xu, L. Li, J.A. Valdez, M. Saber, Y. Zhu, C.C. Koch, R.O. Scattergood, Effect of nanooxide particle size on radiation resistance of iron-chromium alloys, J. Nucl. Mater. 469 (2016) 72-81. https://doi.org/10.1016/j.jnucmat.2015.11.044

[39] T. Stan, Y. Wu, J. Ciston, T. Yamamoto, G.R. Odette, Characterization of polyhedral nanooxides and helium bubbles in an annealed nanostructured ferritic alloy, Acta Mater. 183 (2020) 484-492. https://doi.org/10.1016/j.actamat.2019.10.045 
[40] M.L. Jenkins,M.A Kirk, Characterization of Radiation Damage by Transmission Electron Microscopy, IOP Series in Microscopy in Materials Science, CRC, Boca Raton,2000. https://doi.org/10.1201/9781420034646

[41] B. Yao, D.J. Edwards, R.J. Kurtz, G.R. Odette, T. Yamamoto, Multislice simulation of transmission electron microscopy imaging of helium bubbles in Fe, J. Electron Microsc.

(Tokyo). 61 (2012) 393-400. https://doi.org/10.1093/jmicro/dfs065

[42] Y. Dai, G.R. Odette, T. Yamamoto, The effects of helium in irradiated structural alloys, in:

R.J.M. Konings (Ed.), Comprehensive Nuclear Materials, Vol.1, 2012, Elsevier, Oxford, 2012, pp. 141-193. https://doi.org/10.1016/B978-0-08-056033-5.00006-9

[43] A.D. Brailsford, R. Bullough, The rate theory of swelling due to void growth in irradiated metals, J. Nucl. Mater. 44 (1972) 121-135. https://doi.org/10.1098/rsta.1981.0158.

[44]A.D. Brailsford, R. Bullough, The theory of sink strenght, Philos. Trans. R. Soc. London. Ser. A, Math. Phys. Sci. 302 (1981) 88-136. https://doi.org/10.1098/rsta.1981.0158.

[45] V.A. Borodin, Generalized rate theory for spatially inhomogeneous systems of point defect sinks, Phys. A Stat. Mech. Its Appl. 211 (1994) 279-316. https://doi.org/10.1016/03784371(94)00138-3

[46] V.A. Borodin, Radiation induced swelling, State-of-the-Art Report on Structural Materials Modeling, Rep. NEA/NSC/R(2016)5, OECD, 2016, pp. 165-225.

[47] V.I. Dubinko, New insight on bubble-void transition effects in irradiated materials, J. Nucl. Mater. 206 (1993) 1-21. https://doi.org/10.1016/0022-3115(93)90228-Q

[48] N.W. Ashcroft, N.D. Mermin, Solid State Physics, Harcourt College, Fort Worth,1976. [49:48] N.F. Carnahan, K.E. Starling, Equation of State for Nonattracting Rigid Spheres, J. Chem. Phys. 51 (1969) 635-636. https://doi.org/10.1063/1.1672048

[50] C.A. Walsh, J. Yuan, L.M. Brown, A procedure for measuring the helium density and pressure in nanometre-sized bubbles in irradiated materials using electron-energy-loss spectroscopy, Philos. Mag. A. 80 (2000) 1507-1543.

https://doi.org/10.1080/01418610008212134

[51] G.S. Was, Radiation-Enhanced Diffusion and Defect Reaction Rate Theory, in:

Fundamentals of Radiation Materials Science: Metals and Alloys, Springer, New York, 2017, pp. 207-252. https://doi.org/10.1007/978-1-4939-3438-6_5

[52] E. Kuramoto, T. Tsutsumi, Computer simulation of the bias factor in void swelling in metals, J. Nucl. Mater. 212-215 (1994) 175-178. https://doi.org/10.1016/0022-3115(94)90051-5 [53] S.I. Golubov, A. V Barashev, R.E. Stoller, Radiation Damage Theory, in: R.J.M. Konings (Ed.), Comprehensive Nuclear Materials, Vol. 1, Elsevier, Oxford, 2012, pp. 357-391. https://doi.org/https://doi.org/10.1016/B978-0-08-056033-5.00029-X. 
[54] Y. Iijima, K. Kimura, K. Hirano, Self-diffusion and isotope effect in $\alpha$-iron, Acta Metall. 36 (1988) 2811-2820. https://doi.org/10.1016/0001-6160(88)90127-7. 


\section{List of Tables}

Table 1. Typical microstructural parameters of ODS-Eurofer steel in tempered state

\begin{tabular}{llcc}
\hline Microstructural component & \multicolumn{1}{c}{ Parameters } & Values & Ref. \\
\hline Grain boundaries & Mean grain length & $0.7 \mu \mathrm{m}$ & \\
& Means grain width & $0.3 \mu \mathrm{m}$ & \\
& Volume density & $7.7 \times 10^{-6} \mathrm{~m}^{-1}$ & \\
& Density & $1.3 \times 10^{14} \mathrm{~m}^{-2}$ & {$[12,13]$} \\
Dislocations & Average diameter & $12.0 \mathrm{~nm}^{21}$ & {$[34]$} \\
Oxide nanoparticles & Number density & $10.0 \times 10^{-3}$ & {$[10]$} \\
& & & \\
\hline
\end{tabular}

Table 2. Comparison of average sizes and volume number densities of cavities and swelling contributions for different cavity populations in the ODS-Eurofer steel sample after dual-beam $\mathrm{He}^{+}+\mathrm{Au}^{2+}$ and single-beam $\mathrm{He}^{+}$irradiations

\begin{tabular}{|c|c|c|c|c|c|c|}
\hline \multirow{2}{*}{$\begin{array}{l}\text { Structural } \\
\text { component }\end{array}$} & \multicolumn{3}{|c|}{ Dual beam $\mathrm{He}^{+}+\mathrm{Au}^{2+}$ irradiation } & \multicolumn{3}{|c|}{ Single beam $\mathrm{He}^{+}$implantation } \\
\hline & $D_{\mathrm{c}}{ }^{k}, \mathrm{~nm}$ & $\begin{array}{c}\mathrm{Nv}^{k}{ }^{k} \\
10^{22} \mathrm{~m}^{-3}\end{array}$ & $S^{k}, \%$ & $D_{c}{ }^{k}, \mathbf{n m}$ & $\begin{array}{c}N_{V}{ }^{k} \\
10^{22} \mathrm{~m}^{-3} \\
\end{array}$ & $S^{k}, \%$ \\
\hline $\begin{array}{l}\text { Grain } \\
\text { boundaries }\end{array}$ & $7.1 \pm 1.0$ & $2.4 \pm 0.5$ & $0.46 \pm 0.13$ & $4.8 \pm 0.4$ & $5.3 \pm 1.4$ & $0.31 \pm 0.09$ \\
\hline Dislocations & $6.2 \pm 0.8$ & $1.5 \pm 0.2$ & $0.19 \pm 0.05$ & $4.7 \pm 0.2$ & $2.0 \pm 0.2$ & $0.11 \pm 0.01$ \\
\hline $\mathrm{Y}_{2} \mathrm{O}_{3}$ particles & $\begin{array}{c}8.7 \pm 0.9 \\
\mathbf{1 9 . 6} \pm \mathbf{2 . 0} *\end{array}$ & $1.0 \pm 0.1$ & $\begin{array}{c}0.13 \pm 0.04 \\
\mathbf{3 . 2 8} \pm \mathbf{1 . 0 4} *\end{array}$ & $8.1 \pm 0.8$ & $1.0 \pm 0.1$ & $0.10 \pm 0.03$ \\
\hline Volume & $6.3 \pm 0.8$ & $2.3 \pm 0.6$ & $0.29 \pm 0.10$ & $4.4 \pm 0.2$ & $2.8 \pm 0.5$ & $0.12 \pm 0.02$ \\
\hline Total & & $7.2 \pm 2.0$ & $\begin{array}{c}1.07 \pm 0.36 \\
4.22 \pm 1.44^{*}\end{array}$ & & $11.1 \pm 3.0$ & $0.63 \pm 0.22$ \\
\hline
\end{tabular}

* Assuming that all the cavities associated with nano-oxides would constitute "void" population 
Table 3. Estimated sink strengths for ODS-Eurofer steel samples after single-beam and dualbeam irradiations. For cavities on oxide particles, the calculated effective diameters are also included.

\begin{tabular}{|c|c|c|c|}
\hline \multirow[b]{2}{*}{ Sink type } & \multirow[b]{2}{*}{ Parameter } & \multicolumn{2}{|c|}{ Estimated values } \\
\hline & & $\frac{\text { in single-beam }}{\frac{\text { implanted }}{\underline{\text { sample }}}}$ & $\underline{\text { in double-beam }}$ \\
\hline Grain boundaries & Sink Strength, $10^{14} \mathrm{~m}^{-2}$ & 2.6 & 2.6 \\
\hline Dislocations & Sink Strength, $10^{14} \mathrm{~m}^{-2}$ & $2.1 / 2.7^{\mathrm{a}}$ & $2.1 / 2.7^{\mathrm{a}}$ \\
\hline $\begin{array}{l}\text { Cavities in the } \\
\text { bulk }\end{array}$ & Sink Strength, $10^{14} \mathrm{~m}^{-2}$ & 5.8 & 6.7 \\
\hline $\begin{array}{l}\text { Cavities on } \mathrm{Y}_{2} \mathrm{O}_{3} \\
\text { particles }\end{array}$ & $\begin{array}{l}\text { Effective diameter, } \mathrm{nm} \\
\text { Sink Strength, } 10^{14} \mathrm{~m}^{-2}\end{array}$ & $\begin{array}{l}14.5 \\
9.10\end{array}$ & $\begin{array}{l}14.8\left(22.9^{b}\right) \\
9.3\left(14.4^{b}\right)\end{array}$ \\
\hline \multicolumn{2}{|c|}{ Total sink strengths, $10^{14} \mathrm{~m}^{-2}$} & $19.61 / 20.2^{\mathrm{a}}$ & $\begin{array}{c}20.7 / 21.4^{\mathrm{a}} \\
25.8 / 26.5^{\mathrm{a}, \mathrm{b}} \\
\end{array}$ \\
\hline
\end{tabular}

\footnotetext{
${ }^{a}$ The first number stands for vacancies and the second one - for interstitial atoms

${ }^{b}$ In a case where all of the cavities associated with nano-oxides would transform into voids
} 


\section{Figure captions}

Fig. 1. Damage and implanted helium profiles for (a) simultaneous in situ dual beam irradiation regime with $\mathrm{He}^{+}$and $\mathrm{Au}^{2+}$ ions, and (b) single beam irradiation regime with $\mathrm{He}^{+}$ions only, as calculated using SRIM. The achieved total He content and irradiation dose are indicated in the legends.

Fig. 2.(a) HRTEM image of a He filled cavity attached to yttria nanoparticle in ODS-Eurofer steel irradiated with a single $\mathrm{He}^{+}$ion beam to $\sim 8.5 \times 10^{3}$ appm He. HRTEM imaging conditions: $\sim 0.3 \mu \mathrm{m}$ underfocus. (b) Schematic representation of a lens-shaped cavity attached to spherical nanoparticle.

Fig.3. Microstructure of ODS-Eurofer steel after irradiation at $823 \mathrm{~K}$ in (a,c) simultaneous dualbeam regime with $\mathrm{He}^{+}$and $\mathrm{Au}^{2+}$ ions and $(\mathrm{b}, \mathrm{d})$ single-beam regime with $\mathrm{He}^{+}$ions. $\mathrm{BF}$ TEM imaging conditions: $\sim 0.5 \mu \mathrm{m}$ underfocus

Fig.4. Affiliation of cavities with $\mathrm{Y}_{2} \mathrm{O}_{3}$ nanoparticles in ODS-Eurofer steel after simultaneous in situ dual beam implantation with $\mathrm{He}^{+}$and $\mathrm{Au}^{2+}$ ions at $823 \mathrm{~K}$. (a) A large cavity and (b) ordinary cavities. BF TEM imaging conditions: $\sim 0.5 \mu \mathrm{m}$ underfocus.

Fig. 5.The observed bubble sizes on yttria nanoparticles vs. nanoparticle sizes in the ODSEurofer steel sample after dual-beam $\mathrm{He}^{+}+\mathrm{Au}^{2+}$ ion irradiation (filled triangles) and single-beam $\mathrm{He}^{+}$ion implantation (open triangles). Error bars reflect $10 \%$ uncertainties associated with the size of the first Fresnel fringe on bubbles in underfocused images [40,41]. Also shown are the best power law fits for double-beam irradiation (red and black dot lines for 'void' and 'bubble' populations) and single-beam ion implantation (blue solid line). Gray shaded area corresponds to cavities with the size less than the size of host oxide particle $\left(D_{c}^{p} \leq D_{p}\right)$.

Fig. 6. Comparison of (a) cavity mean sizes, (b) the volumetric number densities of cavities, and (c) the impact on swelling for different cavity families in ODS-EUROFER samples irradiated at $823 \mathrm{~K}$ in double beam (red bars) and single-beam (gray bars) regimes. Panel (d) compares the estimated cumulative swelling for the single-beam implanted sample and for two limiting cases (see main text) for the double-beam irradiated sample. 
Fig. 7. The dependence of function $Q$ on normalized cavity radius $\rho$ at different values of normalized gas content in the cavity $\eta_{g}$. (a) $\eta_{g}=0$; (b) $\eta_{g}=0.1$; (c) $\eta_{g}=0.5$; (d) $\eta_{g}=1.0$; (e) $\eta_{g}$ $=1.5$.

Fig. 8. Variation of the factor $f_{l}=n_{g l}^{*} / n_{g}^{*}$ as a function of wetting angle $\alpha$.

Fig. 9. The normalized bubble volume as a function of the cavity to particle size ratio $\zeta$. Different curve colors correspond to different wetting angles as specified in the legend.

Fig. 10. The dependence of $\chi$ on the cavity to particle size ratio $\zeta$. Different curve colours correspond to different wetting angles as specified in the legend.

Fig. 11. The comparison of exact (solid lines) and approximate (dashed lines) values of (a) $\rho_{p}^{*}$ and (b) $\eta_{g p}^{*}$ as a function of the normalized particle size $R_{p} / R^{*}$ at different $\alpha$. Curve colours in both panels are specified in the legend.

Fig. 12. The critical parameters $\rho_{p}^{*}$ (left column) and $\eta_{g p}^{*}$ (right column) as a function of the normalized particle size $R_{p} / R^{*}$ at different $\alpha$ (as indicated in the legends). Curves within each panel correspond either to the ideal gas law (solid) or to HSEOS with $s=1$ (dash), 5 (das-dot) and 10 (dash-dot-dot). 


\section{List of Figures}
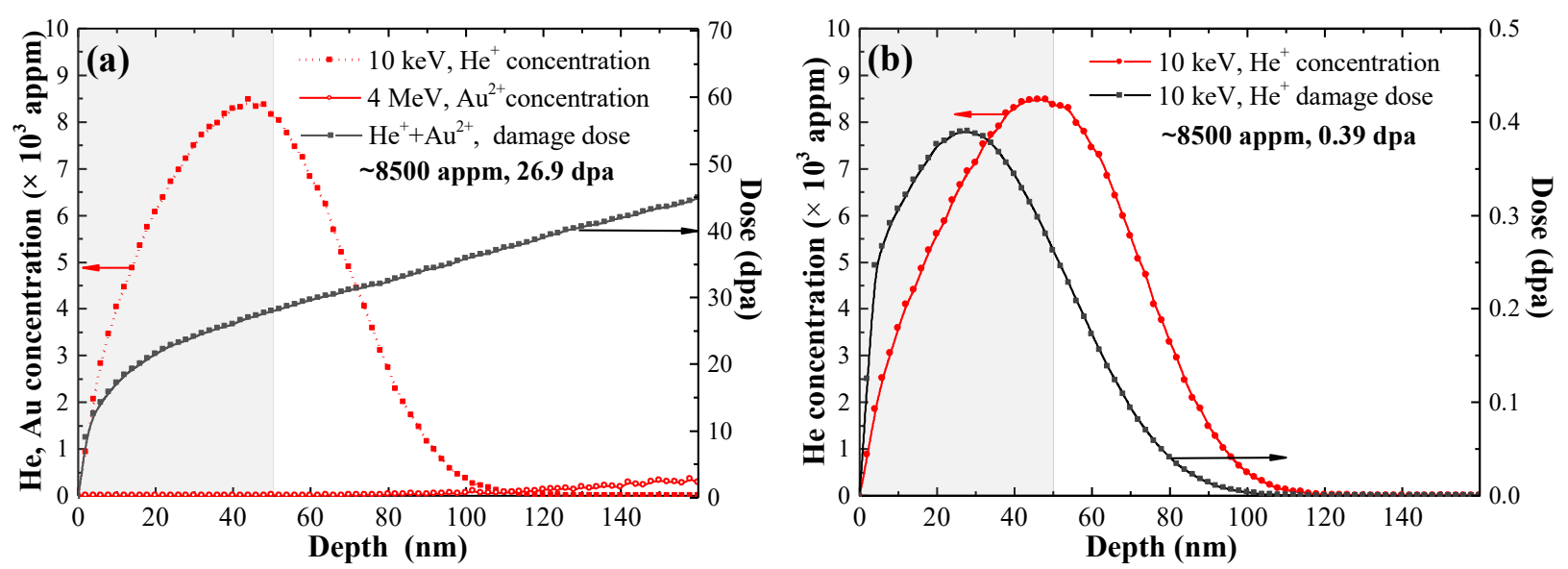

Figure 1
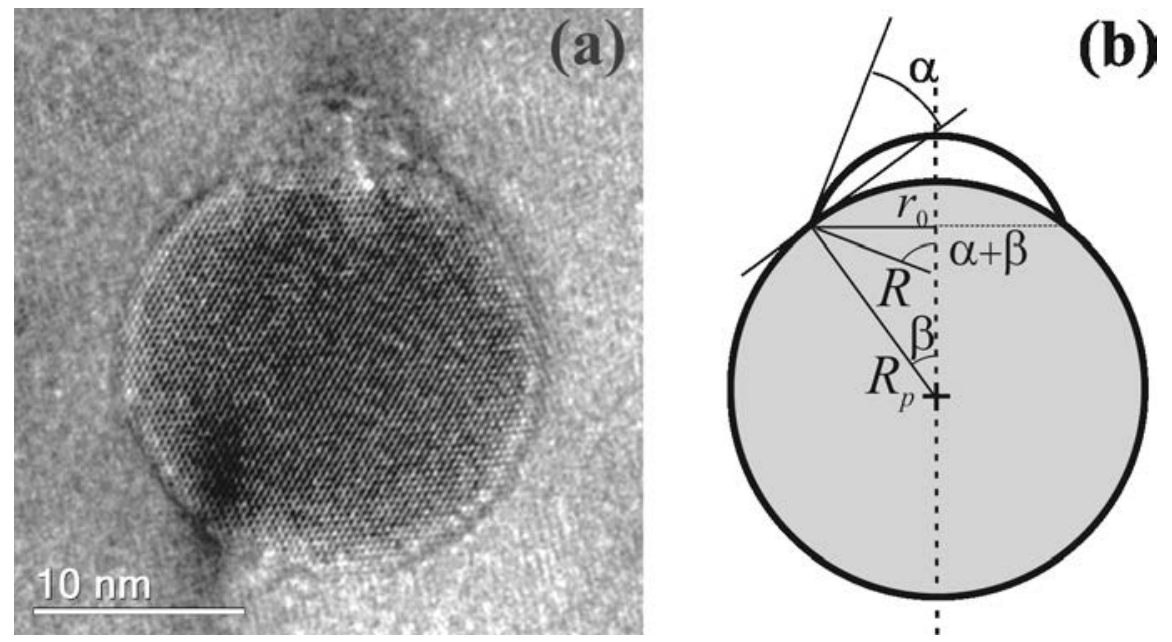

Figure 2 

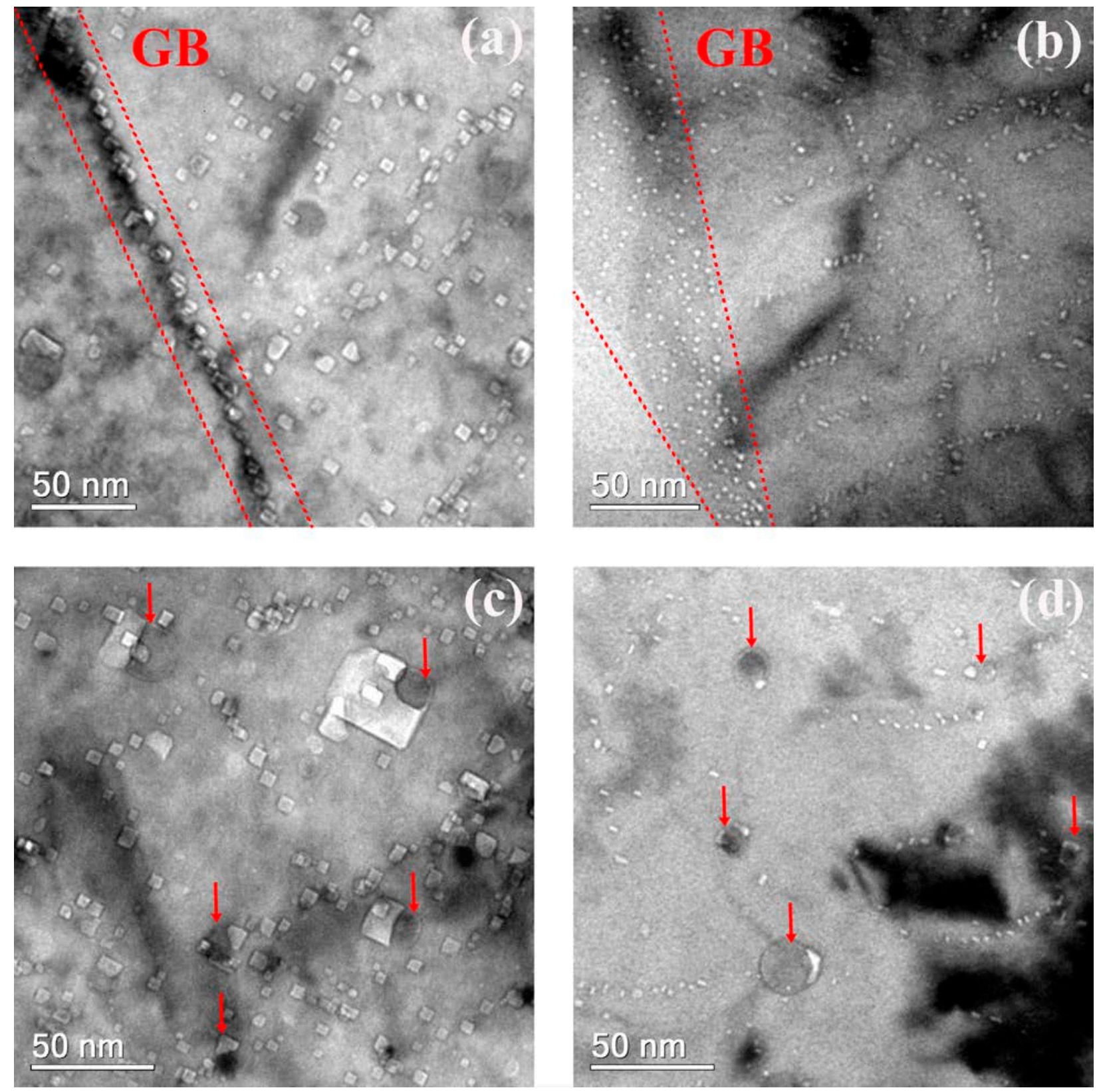

Figure 3 

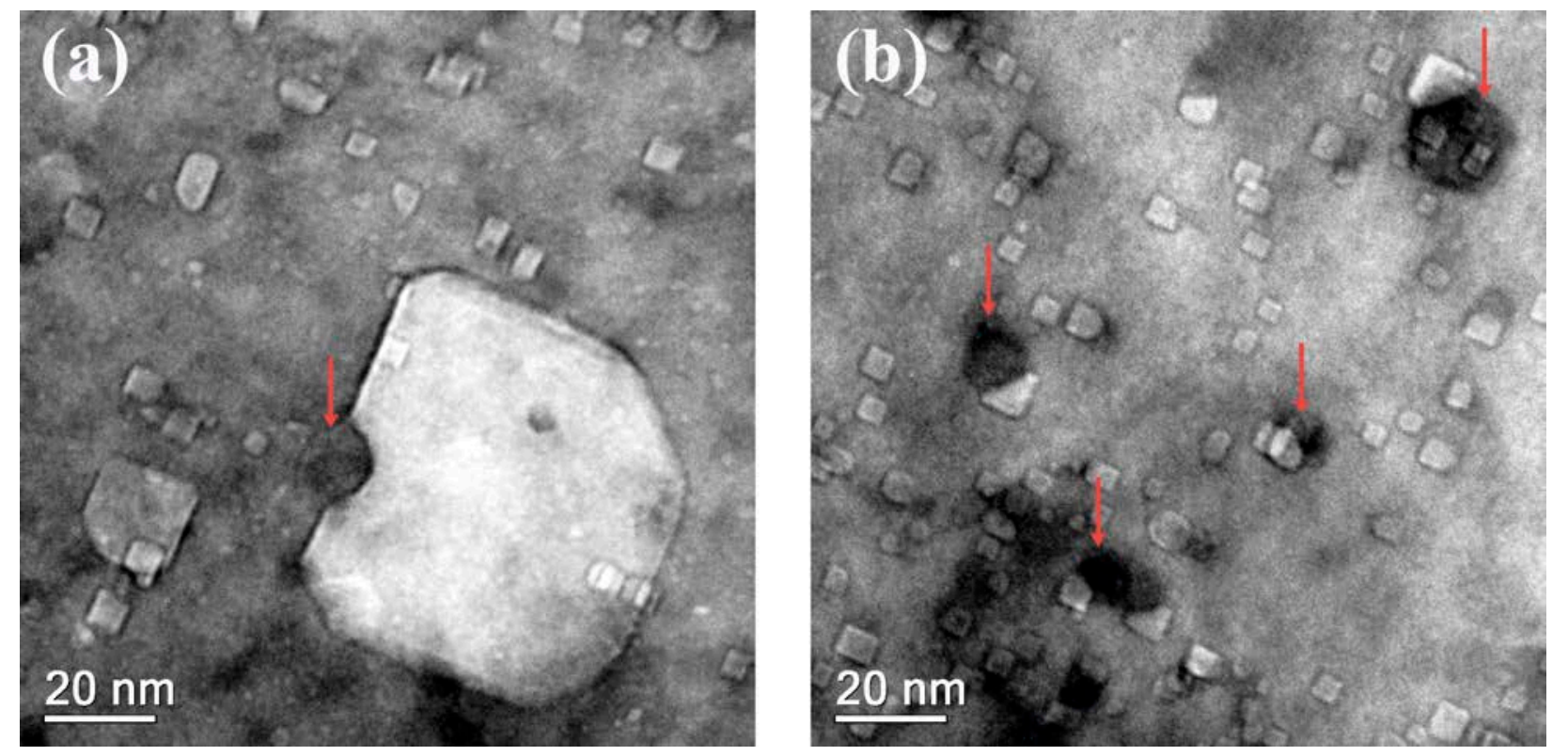

Figure 4

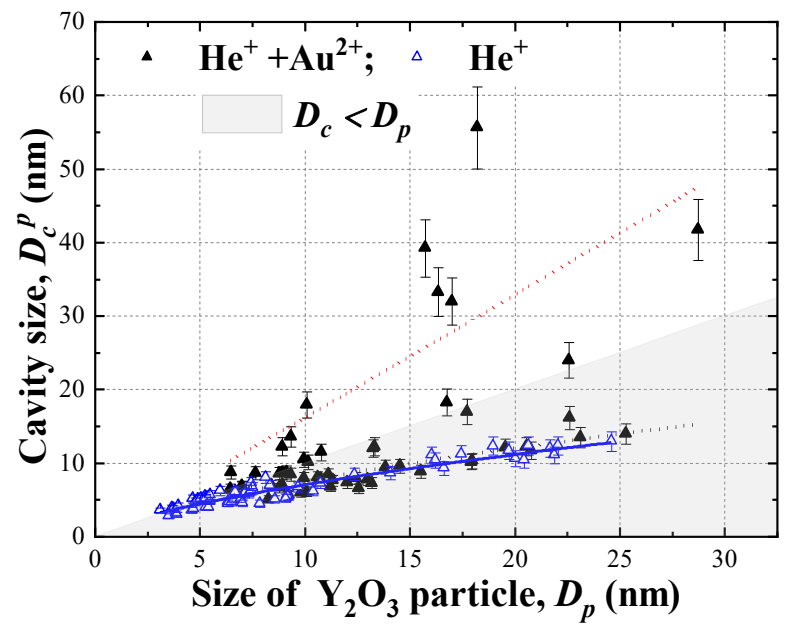

Figure 5 

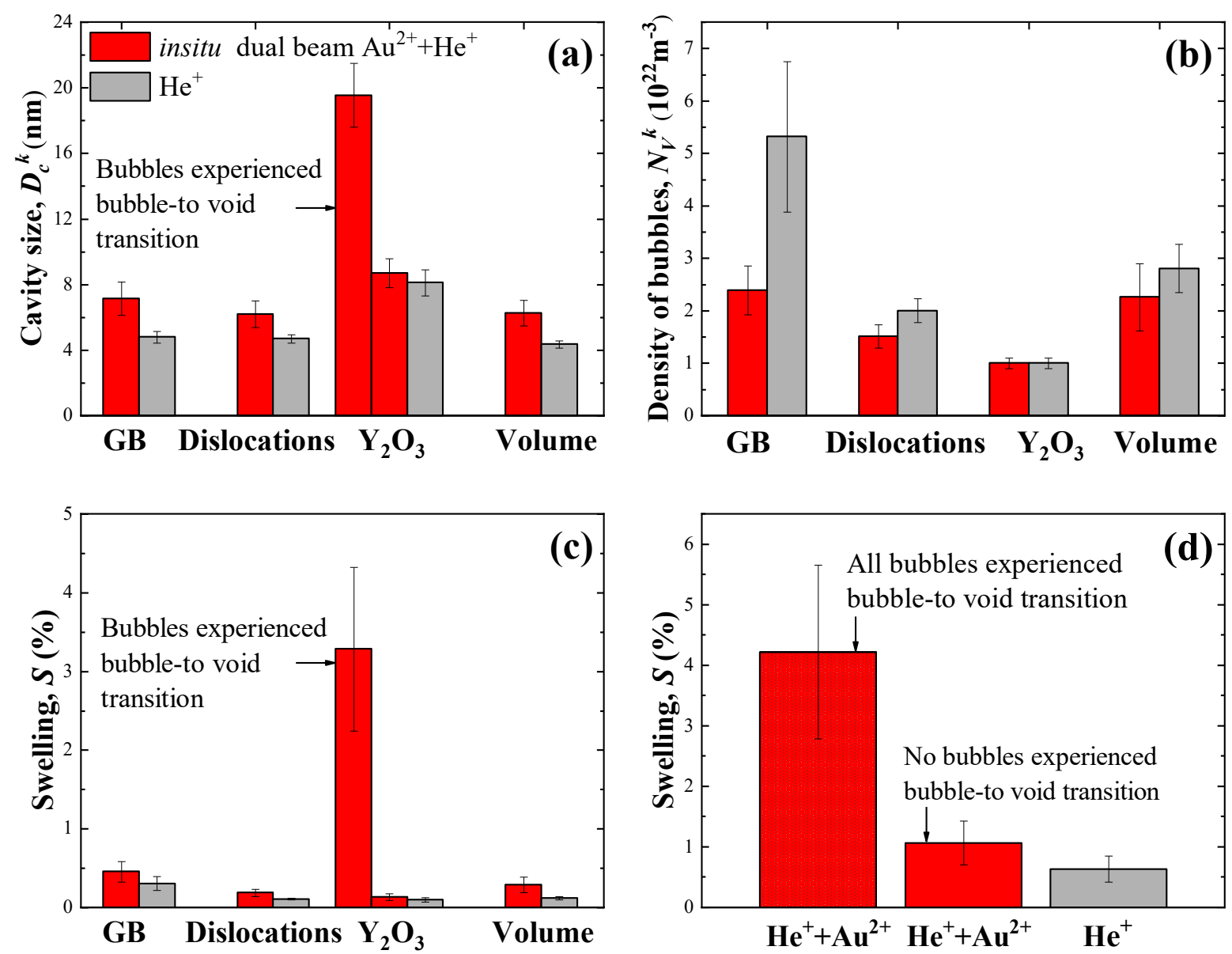

Figure6

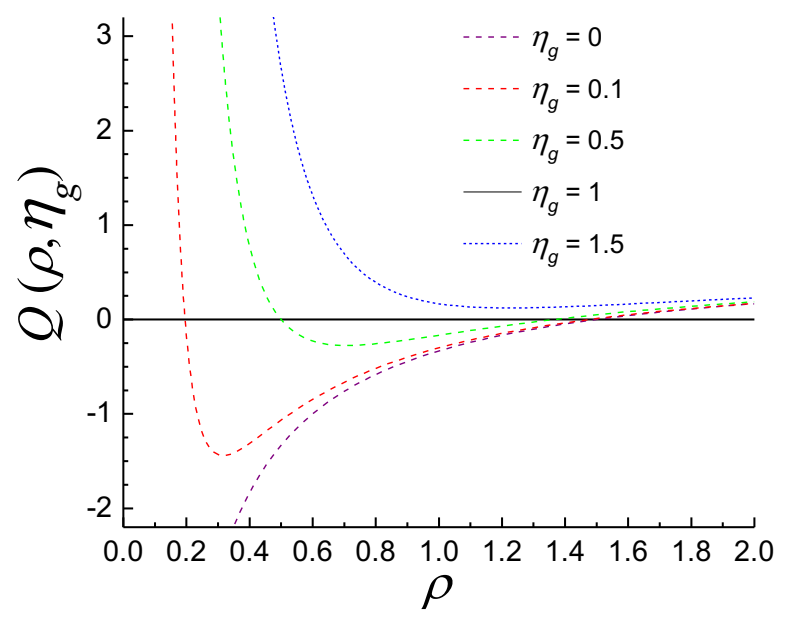

Figure7 


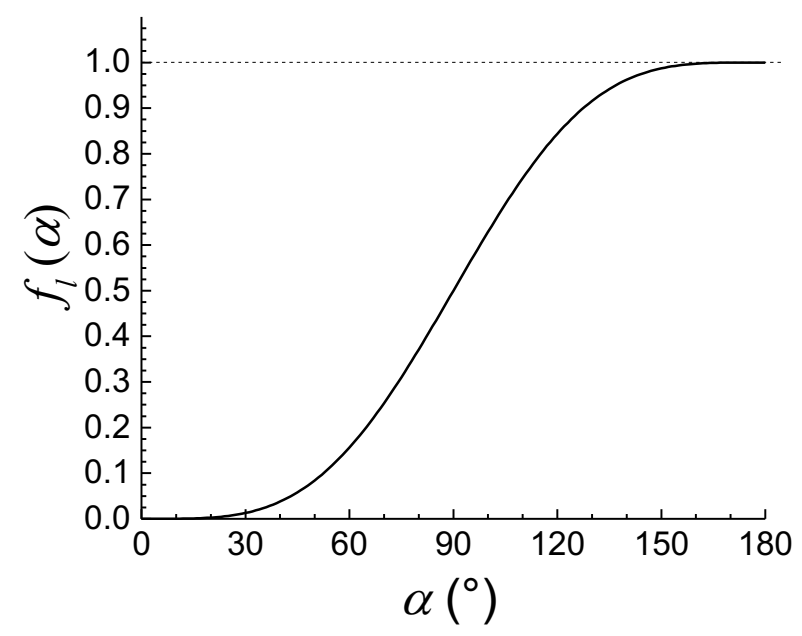

Figure 8

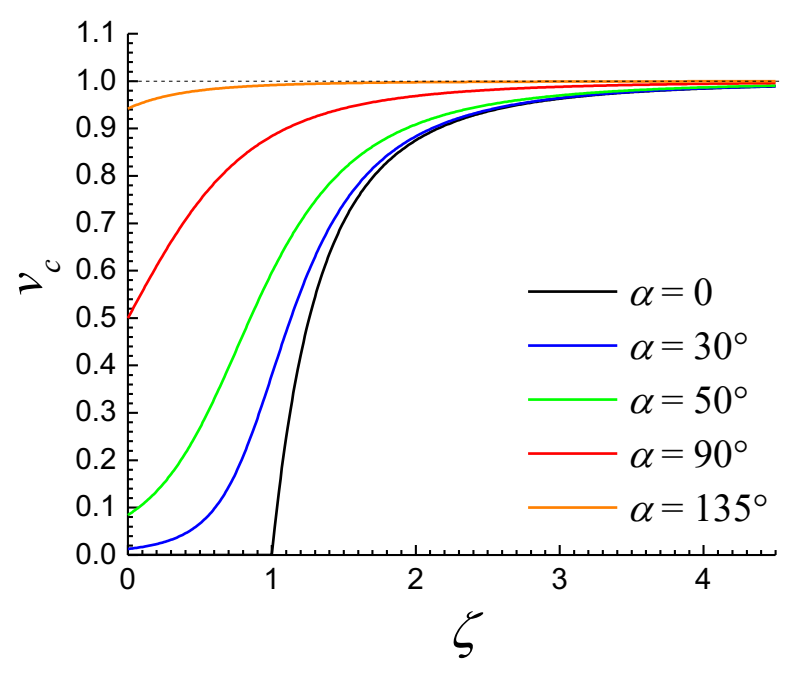

Figure 9

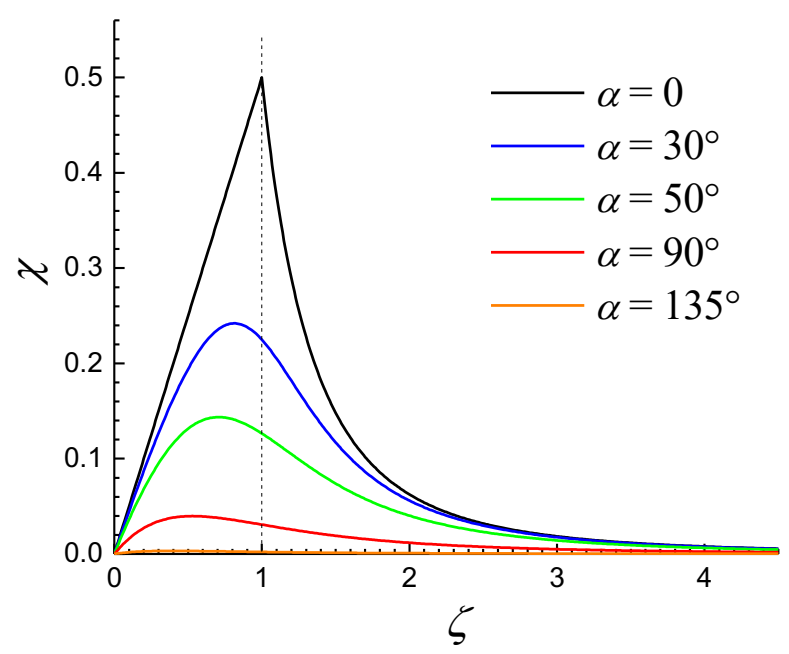

Figure 10 

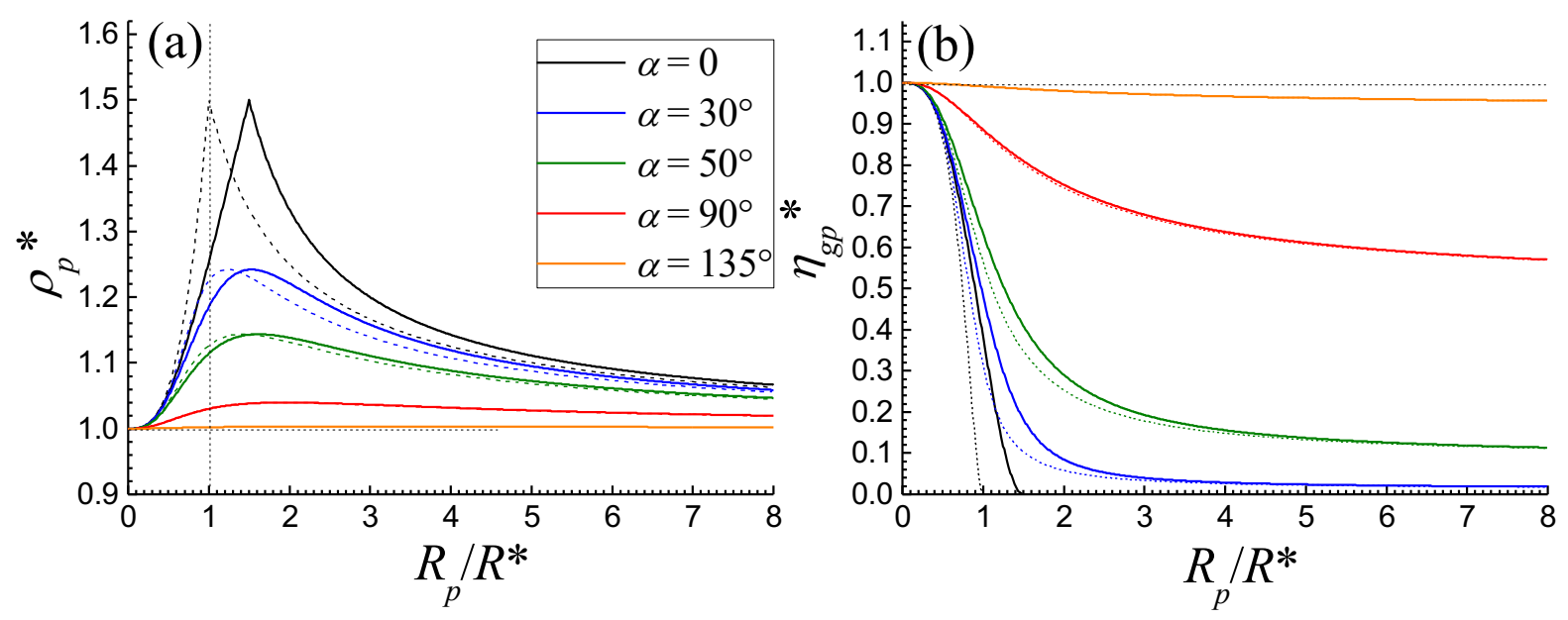

Figure 11 

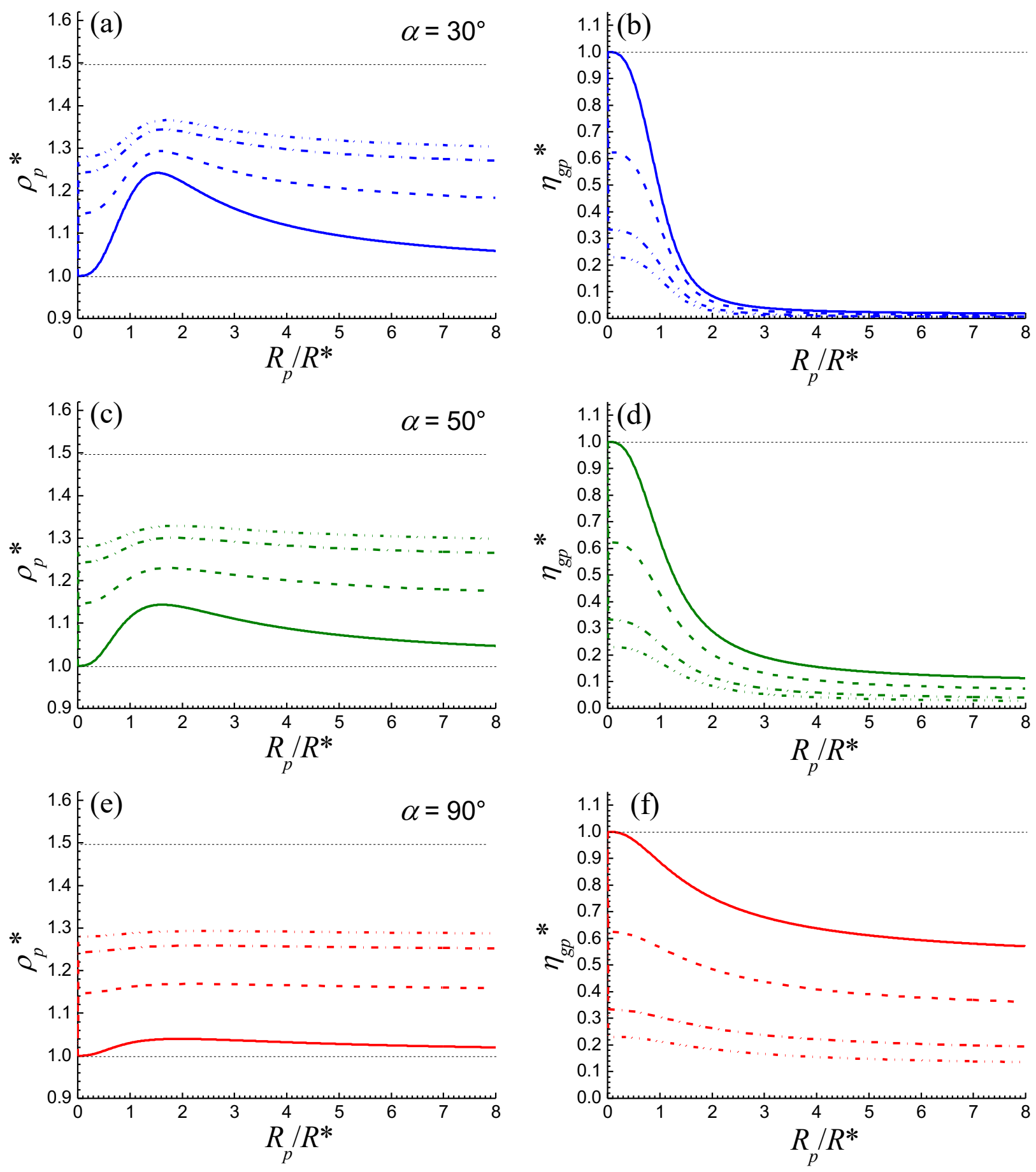

Figure 12 